\title{
Schistosomiasis Associated Glomerulopathy (Glomerulonephristis / Nephrotic Syndrome): A review and Update of the Literature
}

Anthony Kodzo-Grey Venyo

North Manchester General Hospital Department of Urology Manchester United Kingdom.

*Corresponding Author: Anthony Kodzo-Grey Venyo, North Manchester General Hospital Department of Urology Manchester United Kingdom.

Received date: September 24, 2020; Accepted date: December 10, 2020; Published date: December 14, 2020

Citation: Anthony K-G Venyo. (2020) Schistosomiasis Associated Glomerulopathy (Glomerulonephristis / Nephrotic Syndrome): A review and Update of the Literature. J General medicine and Clinical Practice. 3(4) DOI: 10.31579/2639-4162/037

Copyright: @2020 Anthony Kodzo-Grey Venyo. This is an open-access article distributed under the terms of the Creative Commons Attribution License, which permits unrestricted use, distribution, and reproduction in any medium, provided the original author and source are credited.

\section{Abstract}

Schistosomiasis may affect a number of organs within the human body. Schistosomiasis may also be associated with glomerular disease of the kidney in the form of glomerulonephritis and nephrotic syndrome. The association between Schistosomiasis and glomerulopathy may not be well known by a number of practitioners and its pathophysiology may not be very well understood and this could be due to the underdiagnosis of the disease due to the possibility of lack of facilities within the Schistosomiasis endemic areas of the world especially within tropical Africa. Nevertheless, there are a number of patterns of renal involvement in Schistosomiasis which include (a) upper urinary tract sequelae of lower urinary tract Schistosomiasis pathology, (b) immune-related glomerulonephritis, (c) as well as oxidant-stress-mediated renal tubular glomerulonephritis. The renal involvement by Schistosomiasis does tend to be ensued by the development of Schistosomiasis-associated Glomerulopathy (Glomerulonephritis / Nephrotic Syndrome) of varying severity. Individuals affected by Schistosomiasis-associated kidney disease may develop (a) asymptomatic disease which tends to related to self-limited and asymptomatic glomerular disease (b) symptomatic disease which most commonly would tend to present with nephrotic syndrome most often in patients who develop hepato-splenic schistosomiasis with liver fibrosis associated with Schistosoma mansoni infection. Symptomatic patients tend to develop severe hypo-proteinemia, half of the patients tend to have elevated blood pressure. In the absence of nephrotic syndrome, patients who have Schistosomiasis-associated glomerulopathy may manifest with: (a) isolated non-nephrotic syndrome proteinuria (b) acute glomerulonephritis associated with haematuria and heavy proteinuria, (c) Nephrotic syndrome together with systemic manifestations of co-infection with salmonella (class II) or hepatitis C virus (Class I), (d) End stage renal disease (ESRD). Some of the patients who have Schistosomiasis of the kidney may present with Haematuria, Hypertension, Hepato-splenic Schistosomiasis. The diagnosis should be suspected with regard to the following scenarios: (a) clinical suspicion in a patient with kidney disease who is known or has been known to have Schistosoma mansoni; (b) exposure to an endemic area, (c) clinical evidence / demonstration of chronic hepatosplenic schistosomiasis, (d) if the patient has not been diagnosed as having been afflicted with schistosomiasis, then schistosomal infection should be documented, (e) majority of patients with schistosomiasis and kidney disease should have kidney biopsy. (f) The patients should be evaluated for co-infection with salmonella, hepatitis C virus, and hepatitis B virus in order to ensure adequate treatment of the disease. Schistosomiasis is the second most devastating tropical parasitic disease globally which tends to be responsible for many urological complications. Nevertheless, glomerular injury is an uncommon complication which has mainly or most often described with Schistosoma Mansoni. When patients who have Schistosomiasis-associated Kidney disease are managed and followed-up on long-term basis with renal end points, one third of the patients independent of the histopathology examination features of the kidney biopsies would tend to progress dialysis. Membranoproliferative glomerulonephritis (MPGN) is an uncommon complication associated with Schistosoma Haematobium infection which tends to be associated with potentially poor prognosis. MPGN could lead quickly to End Stage Renal Disease (ESRD). Anti-helminthic and immunosuppressive medicaments tend not to be effective at advanced stages of the disease and hence efforts need to be focused upon the prevention, early detection, as well as treatment of Schistosoma infections among at-risk groups of individuals. In order to reduce morbidity related to Schistosoma-associated nephropathy, public health policy, should be concentrated upon the prevention of the disease by the control of snail, improved sanitation, and health education, as well as by the implementation, and sustenance of chemotherapybased control strategies. Considering that many individuals are found yearly to be inflicted by Schistosomiasis who tend to dwell in more rural parts of their countries where facilities for the undertaking of renal function tests and biopsies of the kidney tend not to be readily available, there is the likelihood that Schistosomiasis-associated nephropathies may be highly underdiagnosed globally.

Key Words: schistosomiasis, haematobium; mansoni; japonicum; mekongi; intercalatum; nephrotic syndrome; glomerulonephritis; dialysis; prevention

\section{Introduction}

Schistosomiasis (Bilharziasis) is caused by tremades (blood flukes) of the genius Schistisoma. The WHO fact sheet No 115, [1] has ranked Schistosomiasis as the third most tropical disease globally after malaria and intestinal worms (helminthiasis). The Who fact sheet No 115, [1] also indicated that Schistosomiasis is a major cause of morbidity and mortality in developing countries in Africa, South America, the Caribbean, the Middle East and Asia. [1]Documentation from World Health 
Organization data [1] indicated that more than 207 million people globally have been afflicted with Schistosomiasis and $85 \%$ of the afflicted patients reside in Africa. World Health Organization documentations [1] [2] also estimated that in 76 countries where Schistosomiasis had been regarded as an endemic disease 700 million individuals face the risk for the development of the disease. These documentations additionally indicated that agricultural work, domestic chores and recreational activities had exposed individuals who had developed schistosomiasis to infested water. [1] [2] Chistulo et al. [3] stated that world-wide, 200,000 deaths had been attributed to schistosomiasis yearly. It had been intimated that Schistosomiasis, which is also called bilharziasis, or snail fever, was discovered by Theodore Bilharz, a German Surgeon who had worked in Cairo and that in 1851 Theodore Bilharz identified Schistosoma haematobium as the cause of the disease. [4]] It has been stated that most cases of Schistosomiasis affecting human beings are caused by Schistosoma haematobium, Schistosoma mansoni, and Schistosoma Japonicum; nevertheless, other less common species, including Schistosoma mekongi and Schistosoma intercalatum may also be responsible for systematic human disease of Schistosomiasis. [5] Furthermore, it has been iterated that other types of Schistosoma organisms with avian or mammalian primary hosts may result in the development of severe dermatitis by human beings for example, swimmers itch which ensues Trichobilharzia coellata. Most clinicians would be aware of the fact that Schistosomiasis haematobium tends to present with haematuria and that carcinoma of the urinary bladder tends to manifest many years after the patient had had initial symptoms related to Schistosomiasis. Generally clinicians would be aware of the common causes of glomerulonephritis and nephrotic syndrome. In view of the fact schistosomiasis is not on the top list of causes of nephropathy a number clinicians could easily overlook the possibility of schistosomiasis being associated with nephropathy. The ensuing literature review on schistosomiasis is divided into two parts: (A) An overview which has summarized various aspects of Schistosomiasis in general; and (B) which contains miscellaneous narrations and discussions from a number of reported cases, case series and reviews related to Schistosomiasis associated glomerulopathy (glomerulonephritis and nephrotic syndrome)

\section{Method}

Internet data bases were searched including: Google; Google scholar, Yahoo, Bing, Research gate, and PUBMED. The search words that were used included: Schistosomiasis; Schistosoma Haematobium; Schistosoma Mansoni; Schistosoma Japonicum; Schistosoma Intercalatum; Schistosoma Mekongi, Schistosoma glomerulopathy; Schistosoma nephropathy; Schistosoma glomerulonephritis; Schistosoma nephrotic syndrome; Bilharziasis nephropathy; Bilharziasis glomerulonephritis; Bilharzia nephrotic syndrome; Schistosomaassociated nephropathy. Ninety two references were identified which were used to write the review and update of the literature on Schistosomiasis-associated nephropathy which has been divided into two parts: (A) Overview of Schistosomiasis in general and Schistosomiasis nephropathy and (B) Miscellaneous narrations from some case reports, case series and studies related to Schistosomiasis-associated nephropathy/nephrotic syndrome.

\section{Review and Update of Literature.}

\section{(A) Overview}

General characteristics of Schistosomiasis

The pathological process that is associated with Schistosomiasis (Bilharziasis) is related to the immunohistological reactions of the patient to Schistosoma eggs which have been trapped in the tissues of the patient. [5] It has been stated that:

- Antigens which have been released from the Schistosoma egg induce a granulomatous reaction which involve $\mathrm{T}$ cells, macrophages and eosinophils which emanates in clinical disease. [5]

- The determinants of the symptoms and signs of Schistosomiasis include the number and the location of the eggs that are trapped within the tissues. [5]

- In the first instance the inflammatory reaction is easily reversible but in the subsequent stages of the disease.

- The pathology of schistosomiasis is related to the deposition of collagen and fibrosis, which ensues in damage to the organ involved by schistosomiasis which may only be partially reversible.

A number of authors [6] [7] [8] had iterated that:

- Schistosoma eggs can end up in various organs including: the skin, brain, adrenal glands, muscle, and eyes.

- As the eggs penetrate the urinary tract, they are able to find their way into the female genital tract region and then they are able to form granulomas within the uterus, fallopian tube, and ovary.

- Involvement of the central nervous system (CNS) emanates as a sequel of embolization of Schistosoma eggs via the portal mesenteric system into the brain and the spinal cord through the paravertebral venous plexus.

Snail hosts

Different types of snails serve as the intermediate hosts for the various types of Schistosomiasis causing organisms as follows which tend to divided into urogenital Schistosomiasis and intestinal Schistosomiasis causing snail hosts:

Urogenital Schistosomiasis

- Schistosoma haematobium - Bulinus Truncatus species. In Ghana and elsewhere in West Africa Bulinus Trncatus Rohlfsi (Clessin) is an important snail host of Schistosoma Haematobium. [9] complete the subtypes. Schistosoma Haematobium tends to be found in Africa, the Middle East, Corsica (France). [10] Schistosoma haematobium is stated to be endemic in more than 50 countries in Africa and the Middle East and it is also stated to be occasionally seen in Western Asia. The World Health Organization considers it a significant public health problem in much of Africa. Cases of Schistosoma Haematobium have also been found in various areas of Egypt and Sudan (North and South).

Intestinal Schistosomiasis

- Schistosoma mansoni - Biomphelaria (Pfeiferi in Senegal, Umgani valley - (Natal), South Africa and other places. Schistosoma Mansoni generally tend to be found in Africa, the Middle East, the Caribbean, Brazil, Venezuela, and Suriname. [10]

- Schistosoma Japonicum - Oncomelania Schistosoma Japonicum has been found in China, Indonesia, and the Philippines. Schistosoma Japonicum that was found in Japan has apparently been eradicated in Japan.

- Schistosoma mekongi - Neotricula asperta Schistosoma Mekongi has been found in Several districts of Cambodia, and the Lao People's Democratic Republic. [10]

- Schistosoma guineensis and related Intercalatum - Bolinus. Rain forest areas of Central Africa. [10]

\section{Complications}


Various complications and presentations may be encountered in patients with Schistosomiasis depending upon the type of schistosomiasis and the organ involved. Some of the findings and complications include [5]:

- Bleeding from the gastrointestinal tract

- Obstruction of the gastro-intestinal tract

- Malnutrition

- Schistosoma nephropathy

- Renal failure

- Pyelonephritis

- Visible Haematuria with or without clots

- Haematospermia

- $\quad$ Squamous cell carcinoma (for example of the urinary bladder)

- Sepsis (Salmonella)

- Pulmonary arterial hypertension is a complication which has been reported to occur in approximately $7.7 \%$ of patients who had been affected by hepatosplenic Schistosomiasis in Schistosoma mansoni and Schistosoma Japonicum and perhaps Schistosomiasis mekongi infections. [11] The prevalence of this disease globally has been estimated to be in excess of 270,000 individuals. [11]

- Cor pulmonale

- Neuroschistosomiasis including transverse myelitis, paralysis, and central micro-infarcts. Walker and Zunt [12] stated in view of the smaller size of the egg of Schistosoma Japonicum, S Japonicum accounts for $60 \%$ of all Schistosoma brain infections, with central nervous system which occur in between $2 \%$ and $4 \%$ of all Schistosoma Japonicum infections. Wan et al. [13] intimated that one million people in China had been estimated to be afflicted by Schistosoma Japonicum and that nodular enhancing cerebellar lesions can also occur in Schistosoma Japonicum infections. Furthermore, Walker and Zunt [12] stated that central nervous system schistosomiasis can also occur in patients afflicted with other species of schistosomiasis and that spinal schistosomiasis as a result of Schistosoma mansoni infection tend to manifest as transverse myelitis in view of the larger size of schistosoma mansoni. Walker and Zunt [12] additionally stated that Schistosoma haematobium can also infect the brain or spinal cord. Schistosoma mekongi has been confined to the Mekong basin in Laos and Cambodia where about 140,000 people have been estimated to be at risk of developing this infection. Walker and Zunt [12] intimated that neurological symptoms can develop a number of months pursuant to the infection and that Cauda equina syndrome, anterior spinal artery syndrome, and quadriparesis can occur; majority of the lower spinal cord is affected. Houston et al. [7] reported a mass in the temporal region of the brain which caused paraesthesias of the arm, leg as well as dysphasia with schistosoma mekongi infection. Walker and Zunt [12] reported a rare case of cerebellar Schistosomiasis Japonicum that lacked extra-cranial involvement. The clinical symptoms included head ache, dizziness, and nausea. The results of the patient's blood tests were normal and parasite eggs were identified within the stool of the patient.

- Infertility
- Severe anaemia

- Delivery of low-birth weight babies.

- Spontaneous abortion

- Higher risk of spontaneous abortion

- Higher risk of ectopic pregnancies

- End-organ disease

- Portal hypertension

- Obstructive uropathy

- Complications of pregnancy emanating from fallopian tube granuloma or granuloma of vulvar

- Carcinoma of liver

- Carcinoma of Urinary bladder

- Carcinoma of gall bladder

- Stricture of the ureter

\section{Pathophysiology}

Acute Schistosomiasis (Katayama syndrome)

Katayama syndrome which is an acute schistosomiasis is a terminology which describes a systemic serum sickness-like ailment which develops after a number of weeks in some patients but this does not occur in most patients with new schistosoma infections. Ahmed et al. [5] stated that:

- Katayama syndrome may correspond with the first the first cycle of deposition of eggs in the tissues and this tends to be associated with peripheral eosinophilia and circulating complexes.

- Katayama syndrome is most common with Schistosoma japonicum and Schistosoma mansoni infections and it most likely occurs in heavily infected patients after primary infection.

- The symptoms of Katayama syndrome most often subside over a number of weeks, nevertheless; the syndrome could be fatal.

- Early treatment of Katayama syndrome with cidal medicaments might exacerbate the syndrome which may it necessary to employ the concomitant use of glucocorticoid treatment.

- Within hours after the patient had been exposed to cercariae, mild maculopapular skin lesions may develop.

- With regard to the major human Schistosomal pathogens, the development of significant dermatitis is rare, perhaps, the invading and developing cercariae might be minimally immunogenic. Nevertheless; abortive human infection by schistosomiasis species which depend upon other primary hosts might cause marked dermatitis or swimmer's itch. This selflimited process could recur in a more intense form with subsequent exposures to the same species of Schistosoma.

\section{Chronic schistosomiasis}

Ahmed et al. [5] stated that chronic schistosomiasis is more common in comparison with acute schistosomiasis. They also stated that:

- The pathology of chronic schistosomiasis emanates from egginduced immune-response, granuloma formation, and the associated fibrotic changes.

- Even though cercarial and adult worms are mildly immunogenic, Schistosomal eggs are highly / strongly immunogenic and they tend to induce vigorous circulating and local immune responses. The eggs might require an intense immune response to enhance their migration through the body. 
- Adults worms are capable of absorbing host proteins and if they are not attacked by the immune system, they can live for a number of years in the blood stream in view of the fact that they are coated with host antigens.

- Bloody diarrhoea, cramping, and inflammatory colonic polyposis may be caused by egg retention and granuloma formation in the gut wall usually due to Schistosoma mansoni or Schistosoma japonicum.

- An increased rate of recurrent Salmonella infection, generally with positive blood cultures for Salmonella and negative stool cultures for Salmonella had been linked with patients who have had heavy bowel wall involvement of schistosomiasis.

- Some authors had intimated that chronic schistosomiasis of the intestine may manifest with acute complications of appendicitis [14] [15], perforation, and bleeding long after they had travelled and had been exposed to schistosomiasis in an endemic area.

- Perforation of the rectum caused by Schistosoma haematobium had also been reported. [16]

- Schistosoma eggs have the capability of penetrating the bowel adjacent to the mesenteric blood vessels where the adult words had been residing and the unshed eggs which have been swept back into the portal circulation, lodge in the portal circulatory system and induce granulomatous reactions in the portal tracts.

- Hepatic disease is produced as a result of heavy infestations and this eventually could lead to severe peri-portal fibrosis in a typically characteristic pipe-stem pattern also called "Symmers pipestem fibrosis". Peripheral fibrosis can emanate in portal hypertension which may lead to splenomegaly, ascites, oesophageal variceal bleeding, and the development of portosystemic collateral vessels. The eggs can traverse these collaterals (or in the case of schistosomiasis of the urinary bladder through the inferior vena cava) and reach the pulmonary circulation. The ensuing pulmonary granulomatosis and fibrosis may emanate in pulmonary hypertension or frank cor pulmonale with a high mortality rate.

- Lapa et al. [11] reported an $18.5 \%$ incidence of pulmonary hypertension in patients who had been afflicted by hepatosplenic schistosomiasis.

- Co-infection with hepatitis B or hepatitis C can potentiate hepatic dysfunction and accentuate the risk for the development of hepatocellular carcinoma in excess of what is associated with hepatitis alone. Furthermore, carcinoma of the gall bladder may be associated with schistosomal infection.

- The retention of schistosoma egg and granuloma formation within the urinary tract by Schistosoma haematobium may be ensued by haematuria, dysuria, vesical polyps, and ulcers, and even obstructive uropathies. Schistosoma haematobium infection is also linked with a high rate for the development of carcinoma of the urinary bladder, especially, squamous cell carcinoma rather than transitional cell carcinoma. [17]

- It had been documented that deposition of ectopic egg can lead to other clinical syndromes which include: skin involvement, involvement of lungs, brain involvement, involvement of muscles, involvement of adrenal glands, involvement of the genitalia, involvement of the eyes, involvement of the central nervous system which can lead to transverse myelitis (best described in association with Schistosoma haematobium, and Schistosoma mansoni), and or cerebral disease which had been most common with Schistosoma japonicum infection). Furthermore, invasion of local tissue by eggs leads to the release of toxins and enzymes which provokes a TH-2mediated immune response. [18]

\section{Aetiology}

The Center for Disease Control and Prevention has made the ensuing summations related to the causative agents of Schistosomiasis as follows: [19]

Schistosomiasis (Bilharziasis) is stated to be caused by some species of blood trematodes (flukes) in the genus Schistosoma. The three main species that tend to infect human beings include Schistosoma haematobium, Schistosoma Japonicum, and Schistosoma Mansoni. Three other species of Schistosoma exist which tend to be more localized geographically, which include Schistosoma Mekongi, Schistosoma Intercalatum, as Shistosoma Guineensis that was previously considered to be synonymous with Schistosoma intercalatum. It has been iterated that there had also been a few reported cases of hybrid Schistosomes of cattle origin (Schistosoma haematobium, $\mathrm{x}$ Schistosoma bovis, X Schistosoma curassani, x Schistosoma mattheei which had infected human beings. Unlike trematodes, which are hermaphroditic, Schistosoma spp. are said to be dioicous (individuals of separate sexes). It has been furthermore, iterated that other species of Schistosomes exist, which tend to parasitize birds as well as mammals, and they could cause cercarial dermatitis within human beings but this is clinically distinct from Schistosomiasis. 


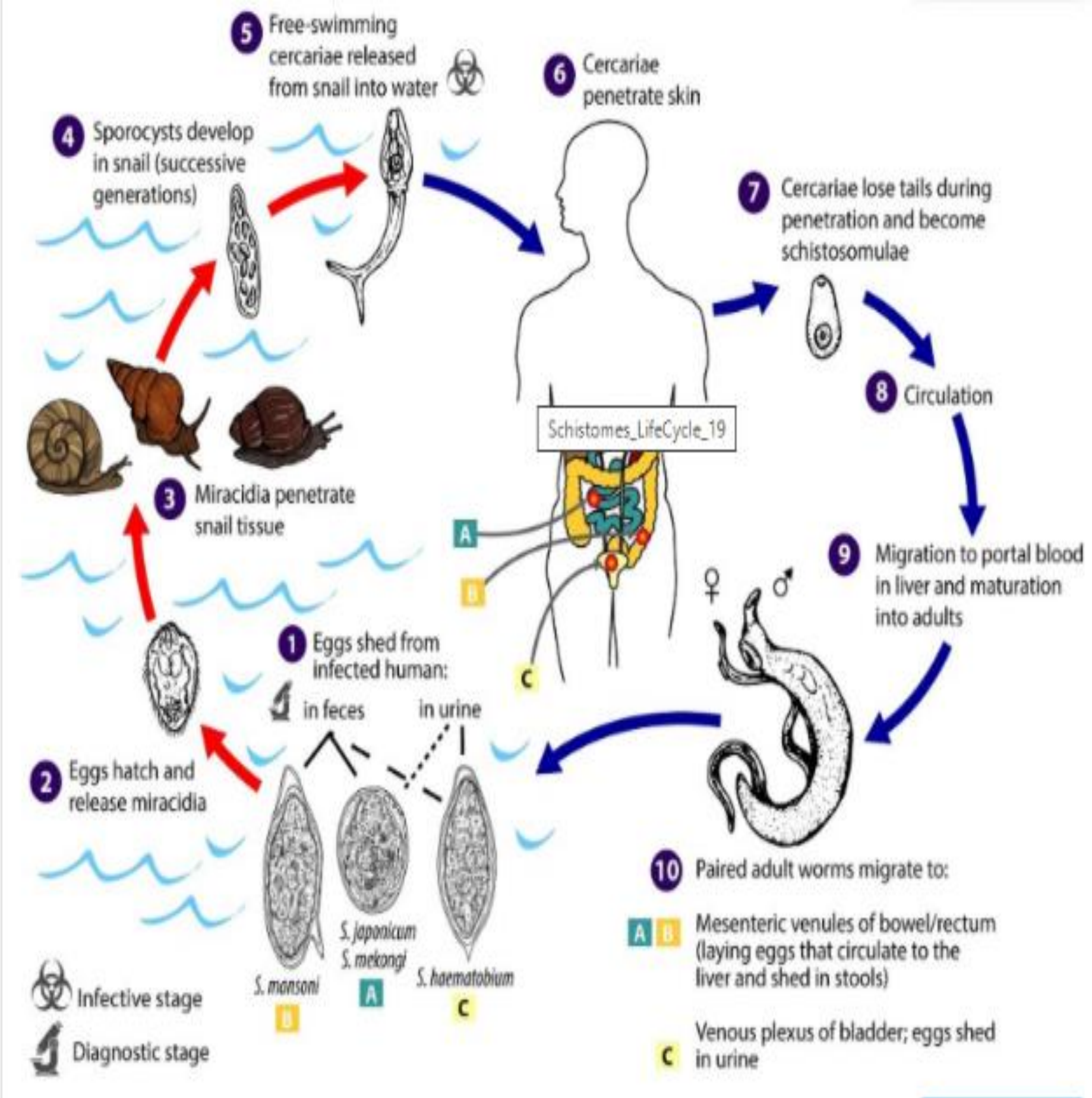

Figure 1:

Life Cycle of Schistosomiasis Reproduced from CDC. And Summated as Follows:

Life cycle -The life cycle of Schistosoma has been described and summated by the Center for Disease Control and Prevention as follows as well as illustrated in figure 1 above.: Schistosoma eggs are eliminated with faeces or urine, depending on species conditions the eggs hatch and release miracidia
Under appropriate , which swim and penetrate specific snail intermediate hosts include two generations of sporocysts

\section{4} . The stages in the snail and the production of cercariae 5 . Upon release from the snail, the infective cercariae swim, penetrate the skin of the human host 6 , and shed their forked tails, becoming schistosomulae 7 . The schistosomulae migrate via venous circulation to lungs, then to the heart, and then develop in the liver, exiting 
the liver via the portal vein system when mature, 89 . Male and female adult worms copulate and reside in the mesenteric venules, the location of which varies by species (with some exceptions) 10 . For instance, $S$. japonicum is more frequently found in the superior mesenteric veins draining the small intestine $\mathbf{A}$, and $S$. mansoni occurs more often in the inferior mesenteric veins draining the large intestine 8 . However, both species can occupy either location and are capable of moving between sites. $S$. intercalatum and $S$. guineensis also inhabit the inferior mesenteric plexus but lower in the bowel than S. mansoni. S. haematobium most often inhabitsin the vesicular and pelvic venous plexus of the bladder $\mathbf{C}$, but it can also be found in the rectal venules. The females (size ranges from 7-28 mm, depending on species) deposit eggs in the small venules of the portal and perivesical systems. The eggs are moved progressively toward the lumen of the intestine (S. mansoni,S. japonicum, S. mekongi, S. intercalatum/guineensis) and of the bladder and ureters ( $S$. haematobium), and are eliminated with faeces or urine, respectively 1 .

Reproduced from [19] CDC

Hosts

CDC has iterated the following: [19]

- Various animals including cattle, dogs, cats, rodents, pigs, horses, and goats, tend to serve as reservoirs for Schistosoma. japonicum, and dogs tend to serve as hosts for Schistosoma. mekongi. Schistosoma. mansoni also tends to be frequently recovered from wild primates within endemic areas but it is considered primarily a human parasite and not a zoonosis.

- Intermediate hosts of the Schistosoma are snails of the genera Biomphalaria, (Schistosoma. mansoni), Oncomelania (Schistosoma. japonicum), Bulinus (Schistosoma. haematobium, Schistosoma. intercalatum, Schistosoma. guineensis). The only known intermediate host for Schistosoma. mekongi is Neotricula aperta.

Geographic Distribution

The CDC has summated the geographic distribution of various Schistosoma organisms as follows: [19]

- Schistosoma mansoni is found primarily across sub-Saharan Africa and some South American countries (Brazil, Venezuela, Suriname) and the Caribbean, with sporadic reports in the Arabian Peninsula.

- Schistosoma. haematobium is found in Africa and pockets of the Middle East.

- Schistosoma japonicum is found in China, the Philippines, and Sulawesi. Despite its name, it has long been eliminated from Japan.

- The other, less common human-infecting species have relatively restricted geographic ranges. S. mekongi occurs focally in parts of Cambodia and Laos. S. intercalatum has only been found in the Democratic Republic of the Congo; $S$. guineensis is found in West Africa. Instances of infections with hybrid/introgressed Schistosoma (S. haematobium x $S$. bovis, x S. curassoni, x S. mattheei) have occurred in Corsica, France, and some West African countries.

\section{Clinical Manifestation}

The CDC has made summating iterations related to the manifestations of various types of Schistosomiasis as follows: [19]

- The presenting symptoms of Schistosomiasis tend not to be caused by the worms themselves but they rather tend to be caused by the body's reaction to the eggs.

- Many Schistosoma infections tend to be asymptomatic.

- A local cutaneous hypersensitivity reaction ensuing skin penetration by cercariae could occur and would tend to appear as small, itchy maculopapular lesions.

- Acute Schistosomiasis (Katayama fever) is a systemic hypersensitivity reaction which could occur weeks pursuant to the initial infection, especially by Schistosoma. mansoni and Schistosoma. japonicum. The manifestations tend to include systemic symptoms/signs that include fever, cough, abdominal pain, diarrhoea, hepatosplenomegaly, as well as eosinophilia.

- On rare occasions, Schistosoma infections could lead to central nervous system lesions. Cerebral granulomatous disease might be caused by ectopic Schistosoma. japonicum eggs within the brain, and granulomatous lesions around ectopic eggs within the spinal cord could occur in cases of Schistosoma. mansoni and Schistosoma. haematobium infections. Continuing Schistosomiasis infection could cause granulomatous reactions and fibrosis within the affected organs (for example within the liver and the spleen) which would tend to be associated with signs/symptoms.

- Pathology examination features that tend to be associated with Schistosoma. mansoni and Schistosoma. japonicum schistosomiasis does include various hepatic complications that tend to involve related to inflammation and granulomatous reactions, and occasional embolic egg granulomas within the brain or spinal cord. Pathology of Schistosoma. haematobium schistosomiasis does include haematuria, scarring, calcification, squamous cell carcinoma, as well as occasional embolic egg granulomas within the brain or the spinal cord.

\section{Epidemiology}

- Schistosomiasis tends to be found within Tropical countries within Africa, the Caribbean, Eastern South America, South East Asia, and in the Middle Eastern countries. Schistosoma Mansoni is stated to be found within some areas of South America and the Caribbean countries, Africa, and within the Middle Eastern Countries. Schistosoma Japonicum has tended to be found within the Farr Eastern countries. Schistosoma Mekongi and Schistosoma Intercalatum tend to be locally within South East Asia and within West Africa respectively. [20]

- $\quad$ Schistosomiasis has been stated to be endemic within about 75 developing countries and that Schistosomiasis does mainly affect people who dwell within rural agricultural as well as periurban areas [20] [21] [22]

- Within Africa Schistosomiasis has been reported in countries including Egypt, Ghana, Zimbabwe, Senegal and other countries.

- Various sub-types of Schistosomiasis have been reported in the United States of America, Asia, as well as other international occurrences have been reported globally.

Estimates of Schistosomiasis Infection 
In 2010, approximately 238 million people were stated to have been infected with Schistosomiasis and out of these, $85 \%$ of the patients were stated to live within Africa [23]. It had also been iterated that an earlier estimate of cases of global Schistosomiasis from 2006, had documented the number of individuals who had been infected with Schistosomiasis was 200 million. [24] It has also been iterated that within many parts of the Schistosomiasis affected areas, Schistosomiasis does infest a large proportion of children who tend to be younger than 14 years of age. It has also been iterated that an estimated 600 million to 700 million individuals globally are at risk for the development of Schistosomiasis in view of the fact that they dwell within countries in which Schistosoma organism is common. [20] It has been documented that in 2012, 249 million had needed therapy in order to prevent the development of Schistosomiasis. Also it iterated that in 2014 it was estimated that the total number of people that required treatment for Schistosomiasis was 258875452 of whom 123329536 that amounted to $47.6 \%$ of people requiring treatment for Schistosomiasis were school age children whose ages were between 5 years and 14 years. In $201491.4 \%$ of the people who were estimated to require treatment for Schistosomiasis were living within the continent of Africa. [25] It has additionally been documented that the aforementioned estimated figures of Schistosomiasis infections would perhaps make Schistosomiasis, the commonest global parasitic infection with malaria perhaps the second most common parasitic global infection and which tend to cause approximately 207 million cases of infection in 2013 based upon the stipulations of some authors. [20] It has furthermore, been iterated that Schistosomiasis is also referred to as snail fever as well as bilharzia [26] which is caused by schistosomes and despite the high incidence of the disease it has been listed as a neglected tropical disease. [20] It was documented in Malaria Fact Sheet No. 94 which was updated in March 2014 that in 2012, malaria caused an estimated 627000 deaths with an uncertainty range of 473000 to 789000 which mostly involved African children. It was additionally documented that based upon the latest estimates that were released in December 2013, there were 207 million cases of malaria in 2012 with an uncertainty range of 135 million to 287 million as well as an estimated 627000 deaths with an uncertainty range of 473000 to 789000 as well as the mortality rates of malaria had fallen by $42 \%$ globally since 2000 , and by $49 \%$ within the WHO African Region. [27] It has been documented that Schistosoma Haematobium which tends to be mainly. responsible for infections of the genitourinary tract does infect more than 112 million individuals yearly within SubSaharan Africa alone. [20] [28] Schistosoma Haematobium has been iterated to be responsible for 32 million cases of dysuria, 10 million cases of hydronephrosis, and 150 million cases of death that emanate from kidney failure each year, which would tend to make Schistosoma Haematobium the World's deadliest Schistosome. [20] [28] Considering the large number of cases of various types of Schistosoma infections that are encountered globally each year and the few cases of kidney biopsies that tend to be undertaken in rural parts of the world where Schistosomiasis tend to be more commonly encountered, there is the likelihood that many cases of Schistosoma infection-associated nephropathy could be missed or underdiagnosed.

\section{Deaths related to Schistosomiasis}

It has been documented that estimates related to the number of deaths related to Schistosomiasis have varied. [20] It has been iterated that globally, the Global burden of Disease Study in 2010 had estimated deaths directed related to the disease at 12,000 while the World Health Organization (WHO) in 2014, had estimated greater than 200,000 annual deaths that had been related to Schistosomiasis. [20] [29] It has also been documented that another 20 million individuals had developed severe consequences related to Schistosomiasis. It has also been stated that the estimates of death related to Schistosomiasis do need to be reassessed in view of the fact that the death rate has varied between 24,072 and 200,000 globally each year. In 2000, the World Health Organization did estimate the annual death rate of Schistosomiasis as 200,000 globally and this is expected to have decreased considerably in view of the impact of a scaleup in large chemotherapy campaigns over the preceding decade. It has been documented that the control of Schistosomiasis has been successfully implemented over the preceding 40 years in many countries with the inclusion of Brazil, Cambodia, China, Egypt, Mauritius, Islamic Republic of Iran, Oman, Jordan, Saudi Arabia, Morocco, Tunisia and other countries. It has also been documented that in Burundi, Burkina Faso, Ghana, Niger, Rwanda, Sierra Leone, the United Republic of Tanzania, and Yemen, there has been the possibility to scale-up the treatment of Schistosomiasis to the national level and this has had an impact on the disease over a few years. It has been recommended that an assessment of the status of transmission of Schistosomiasis is needed in many countries. [10] It has also been iterated that Schistosomiasis, is the deadliest of the neglected tropical diseases. [20]

\section{History of Schistosomiasis}

- Schistosomiasis is also by terminology been referred to as bilharzia or bilharziosis within many countries after the German physician Theodor Bilharz, who was the first person to describe the cause of Schistosomiasis of the urinary tract in 1851 . He was a German Pathologist who was working in Egypt in 1851 who described the worm. [20] [30]

- It has been iterated that the first physician who had described the entire Schistosomiasis disease cycle was the Brazilian parasitologist called Pirajá da Silva in 1908. [31] [32] It has been documented that the first known case of Schistosomiasis was discovered in 2014 , which belonged to a child who had lived 6,200 years ago. [33] It has been stated that Schistosomiasis was a common cause of death for Egyptians within the Greco-Roman period. [34]

- It was also documented that in 2016, more than 200 million individuals needed treatment for Schistosomiasis; nevertheless, 88 million people had actually been treated for Schistosomiasis. [20] The WHO documented that estimates had shown that at least 290.8 million individuals did require preventive treatment for Schistosomiasis in 2018, out of which greater than 97.2 million were reported to have undergone treatment [10]

\section{Race-related demographics}

\section{Ahmed et al. [5] stated that:}

- All human beings are equally susceptible to the development of schistosomiasis if they are exposed to infested fresh water. However, the frequency of Schistosoma infection among individuals is dependent upon the geographic distribution of schistosomiasis in the different parts of the world.

- The frequency of some complications of schistosomiasis would appear to differ geographically during infection with the same helminth (for example, ascites is more common in the Middle East and in Brazil).

\section{Sex-related demographics}

It has been documented that [5]:

- Schistosomiasis is more common in men in comparison with women, most probably in view of the increased exposure of men to infected water through bathing, swimming, and agricultural activities.

- Schistosoma haematobium has been responsible for genital lesions in $30 \%$ of women who had been infected.

\section{Age-related demographics}

The age-related demographics of schistosomiasis has been summarized as follows [5]:

- The prevalence and severity of schistosomiasis varies with age and that children and the adolescents are most often infected heavily.

- The rates of schistosomiasis infection and severity of the infection might vary with gender-specific activity at all ages. 
- Congenital infections had been defined and schistosomiasis had been detected within the placenta and new borne had been diagnosed with schistosomiasis and thus confirming congenital schistosomiasis. [35] [36]

\section{Treatment}

Two medicaments namely praziquantel and Oxamniquine are available to be utilized for the treatment of Schistosomiasis. [37] The two medicaments have been stated to be considered as equivalent with regard to their efficacy against Schistosoma Mansoni and their safety. [37] It has additionally been iterated that in view of the fact that Praziquantel has lower cost per each treatment, and the fact that Oxaminiquine does tend not to be efficacious against the urogenital form of Schistosomiasis that is caused by Schistosoma Haematobium, generally, Praziquantel has been regarded the first option of treatment for Schistosomiasis. [38] The aim of treatment of Schistosomiasis is to ensure cure of the disease and to prevent the progression of the acute form of Schistosomiasis to the chronic form of Schistosomiasis. It has been strongly recommended that all cases of suspected Schistosomiasis should be treated irrespective of their manifestation in view of the fact that the adult parasite could live within the host for many years. [20] [39] It has been stipulated that Schistosomiasis can be treated by means of the taking orally of a single dose of Praziquantel medicament annually [20] It has been documented that the World Health Organization has developed guidelines pertaining to the community treatment based upon the impact the disease tends to have on children within villages in which the disease is common as follows: [20]

- When a village does report that greater than 50 percent of its children have blood within their urines, then every one within the village should receive treatment. [20]

- When $20 \%$ to $50 \%$ of children within a village have blood within their urines, then only school-age children should be treated. [20]

- When less than $20 \%$ of children within a village have symptoms related to Schistosomiasis, then mass Schistosomiasis treatment should not be implemented. [20]

Other possible options of treatment for Schistosomiasis do include: utilization of a combination of praziquantel with metrifonate, artesunate, or mefloquine, or mefloquine. [40] It has been documented that a Cochrane review of the literature had found tentative evidence which had documented that utilization of metrifonate alone was as effective as praziquantel with regard to the treatment of Schistosomiasis. [40] Another medicament, mefloquine, which had previously been utilized with regard to the treatment of as well as prevention of malaria, had been recognized between 2008 and 2009 to be effective as treatment for Schistosomiasis. [41] It has been pointed out that historically, utilization of antimony potassium tartrate had remained the treatment option of choice with regard to the management of Schistosomiasis until praziquantel was developed in the 1980s. [42]

Acute Schistosomiasis (Katayama Syndrome)

Chronic Schistosomiasis

\section{Gastrointestinal Schistosomiasis}

Lapa et al. [11] stated that:

- Peripheral fibrosis which has also been called Symmens pipestem fibrosis constitutes the commonest complication of gastro-intestinal schistosomiasis and this leads to the development of portal hypertension and gastrointestinal haemorrhage.

- Liver failure is not common with the exception of patients who have concomitant chronic hepatitis or cirrhosis.

- With regard to patients afflicted by Schistosoma mansoni, Schistosoma Japonicum and perhaps Schistosoma mekongi between $4 \%$ and $8 \%$ develop hepatosplenic disease.

\section{Schistosomiasis of the urinary tract}

Ahmed et al. [5] stated that schistosomiasis of the urinary tract emanate in renal failure as a sequel of obstructive uropathy, pyelonephritis, or carcinoma of the urinary bladder which tends to occur 10 years to 20 years after the initial infection and furthermore, immune complexes which contain worm antigens may be deposited in the glomeruli which can lead to glomerulonephritis and amyloidosis.

\section{Schistosomiasis of the Female Genital tract}

Mosunjac et al. [43] iterated that Schistosoma haematobium does cause lesions in the lower genital tract of female patients including the cervix, vulva and vagina and that schistosomiasis of the female genital tract has been documented to be a major social and medical problem which may enhance the spread of some sexually transmitted diseases including HIV and human papillomavirus (HPV)

Co-existence of sexually transmitted infection and urogenital schistosomiasis

Leutscher et al. [44] reported their study in which the ensuing observations were made:

- Among women in Madagascar who were afflicted with Schistosoma haematobium infections, 35\% had co-existing sexually transmitted infections including Neisseria gonorrhoea, Chlamydia trachomatis, Mycoplasma genitalium, Trichomonas vaginalis in comparison with $17 \%$ of men.

- They observed this to be more common in in the younger populations aged between 15 years and 24 years in comparison with the older populations.

- The association was found to become stronger with greater parasite burden.

It has been stated that HIV replication and cytokine dysregulation does occur in the presence of schistosomiasis in HIV-positive individuals and that immune restoration syndrome had been described with symptomatic schistosomiasis in patients who had been taking anti-retroviral treatment. [5]

Schistosomiasis in pregnancy

Friedman et al. [45] in 2007, reported an association between schistosomiasis in pregnancy with anaemia and low birth weight.

Summary of salient points specifically related to Schistosomiasis glomerulonephritis and nephrotic syndrome

\section{Epidemiology}

\section{Schistosomiasis glomerular disease}

Barsoum [46] stated that the incidence of Schistosomiasis glomerular disease is not known in view of the fact that most cases of schistosomiasis associated glomerular disease are sub-clinical or resolve spontaneously [46]. Essat et al. [47] [48] stated that in a village in Upper Egypt, where Schistosoma haematobium was introduced because of changing irrigation methods, most of the people who acquired the infection developed selflimited nephrotic range proteinuria with biopsy confirmed mesangioproliferative glomerulonephritis. It has been stated that similar glomerular lesions had also been described with recent schistosoma 
mansoni or schistosoma japonicum infection and they usually resolved following antibiotic treatment. [46] Nevertheless, a number of authors, [49] [50] [51] [52] [53] had reported persistent or progressive glomerular disease in about $10 \%$ to $15 \%$ of patients who had developed hepatic fibrosis due to chronic infection with Schistosoma mansoni and also occasionally. Schistosoma haematobium

\section{Pathogenesis}

A number of observations had been made which would indicate a pathogenic role for the immune response to the Schistosoma parasite in the development of glomerulonephritis in patients affected by the disease as follows:

- Houba [54] described glomerular lesions which were similar to those described in humans which were induced by experimental infection of numerous small and large animals.

- Antibodies against schistosomal antigens were often eluted from the glomeruli of affected animals by Houba [54] and humans by Hoshino-Shimizu et al. [55]

- Nussenzveig et al. [53] identified Schistosoma antigens in the glomeruli by using indirect immunofluorescence (in which fluorescenated anti-schistosomal antibodies were incubated with the renal tissue)

- $\quad$ Essat et al. [47] reported an association between Schistosoma infection and clinical glomerular disease.

Sobh et al. [52] indicated that the glomerular injury which occurs in Schistosomiasis glomerulopathy is a sequel of antigens which are released by Schistosoma worms within the tributaries of the portal vein. Nevertheless, progression to advanced pathological lesions and eventual glomerulosclerosis has been postulated to be associated with a variety of factors which include:

- The suggestion of Barsoum et al. [56] that schistosomiasis has the capability of producing hepatic fibrosis which leads to impairment of the clearance of antigens from the portal blood; including schistosomiasis worms, eggs and food antigens. This in the opinion of van Marck et al. [57] is accentuated by intrahepatic portosystemic shunts associated with fibrosis.

- With regard to postulates relating to the role of $\operatorname{IgA}$, some authors [58] [59] [60] had iterated that schistosomiasis is linked with an increase in the local production of $\operatorname{IgA}$ and that in the later stages of infection, IL-10 tends to induces an extensive / significant increase in the expression of $\operatorname{IgA}$ on circulating Blymphocytes. Furthermore, Barsoum et al. [61] had also intimated that: (a) the circulating immunoglobin profile is characterized by significant augmentation of IgA subsets; (b) the impaired clearance of $\operatorname{IgA}$ as a result of the associated fibrosis of the liver emanates in an increase in circulating $\operatorname{IgA}$; (c) patients who have been afflicted by progressive schistosomal glomerulopathy often have predominance of $\operatorname{IgA}$ depositions in mesangial, sub-endothelial, sub-epithelial, and peri-tubular sites, which would be indicative of a pivotal role for increased circulating $\operatorname{IgA}$.

- Two groups of authors [62] [63] had stated that autoimmunity also does have a role to play, in that, patients who had been infected with schistosomiasis experimental animals infected with schistosomiasis may develop anti-DNA rheumatoid factor, as well as, antiphospholipid antibodies.
- Barsoum et al. [46] had stated that Co-infection with other microbes, especially salmonella and hepatitis B and hepatitis C lead to worsening of the glomerular disease associated with schistosomiasis.

- With regard to genetic factors, some authors had stated that genetics would appear to play an important role in the susceptibility of a patient to schistosomiasis, [20], [64] progression to fibrosis of the liver [65], the initiation of glomerulonephritis [66].

- Barsoum [67] had explained the geographical variations in the prevalence of schistosomal glomerulopathy upon the differences in the strains of schistosomiasis and the severity of infection

Clinical and Pathological presentations of schistosomiasis glomerulopathy [Clinicopathological presentation [clinical manifestations; and co-infections]

The clinical manifestations were summarized by Barsoum et al. [46] as follows:

- $\quad$ Asymptomatic - Self-limited and asymptomatic glomerular disease may be common in patients with schistosomal infection (The actual rate of the disease in the opinion of the authors, can only be known following a research project of patients who are afflicted by schistosomiasis)

- Symptomatic patients with schistosomal associated glomerulopathy most frequently manifest with nephrotic syndrome and the patients tend to have hepato-splenic schistosomiasis with fibrosis of the liver associated with Schistosoma mansoni infection. The patients do have severe hypo-proteinemia, but their serum lipid concentrations would usually tend to be low as a sequel of the associated nutritional deficiency and dysfunction of the liver. The patients also commonly have hypertension which is seen in about half of the patients [68] The patients complement levels as well as the levels of other commonly performed serum tests tend to be normal unless there is salmonella o-infection or hepatitis virus which may be associated with abnormal serological results. In the absence of co-infections about $80 \%$ of the patients with nephrotic syndrome would have class III, IV, or V renal histology.

- In the absence of nephrotic syndrome, patients with schistosomal glomerulopathy may present with: (a) isolated non-nephrotic syndrome proteinuria and their glomerular filtration rate would tend to be normal and these patients also tend to have class I histological renal histology and they also tend to be asymptomatic and their diagnosis is usually made incidentally; (b) acute glomerulonephritis associated with haematuria and heavy proteinuria which has been described in recent onset schistpsoma haematobium infection and they tend to have class I renal histology; (c) Nephrotic syndrome together with systemic manifestations of co-infection with salmonella (class II) or hepatitis C virus (Class I) [69]; (d) End stage renal disease (ESRD).

- Some of the patients who have Schistosomiasis of the kidney may present with

$\circ$ Haematuria 


\section{○ Hypertension \\ - Hepato-splenic Schistosomiasis.}

With regard to outcome, Barsoum [46] stated that majority of patients who are asymptomatic with incidentally diagnosed non-nephrotic proteinuria would recover following anti-parasitic treatment. On the other hand, majority of the symptomatic patients would develop progressive renal disease and end up developing end stage renal disease (ESRD) or they would die as a result of other complications of fibrosis of the liver.

\section{Salmonella co-infection}

With regard to Salmonella infection, the patients tend to have a rapid onset of nephrotic syndrome and an active urine sediment which show red and white cell casts [46]. Barsoum et al. [68] stated that the renal function may be impaired as a sequel of sepsis and hypovolaemia and that the serum complement $\mathrm{C} 3$ tends typically to be reduced as a result of alternative pathway activation by bacterial endotoxin, and the patients might additionally have positive serological tests for syphilis, rheumatoid factor, anti-nuclear antibodies, and anti-DNA antibodies. Barnhill et al. [70] indicated that the disease tends to be reversible with combined antiparasitic and antibiotic treatment.

\section{Histological classification}

Barsoum [46] stated that: (a) The African Association of Nephrology (AFRAN) had approved the classification of schistotomal glomerulopathies into five categories based on the prevailing histopathological lesions [51] and (b) As a result of the increasing incidence of co-infection a sixth category has been proposed to increase the categories of glomerulopathies to six. The categories of glomerulopathies have been summarized as follows:

- The Class 1 category refers to mesangial proliferative glomelunonephritis which results from the deposition of parasitic antigens and antibodies. Patients who are classified into Class 1 category are usually asymptomatic and their diagnosis is usually made incidentally when urinalysis reveals mild proteinuria and or microscopic haematuria. The glomerular filtration rate of patients in this category tends to be normal and the patients also tend to have benign disease with a self -limiting following eradication of the infection.

- In The Class II category, there is an exudative glomerulonephritis and light microscopy reveals neutrophils and monocytes in the glomerulus and immunofluorescence microscopy shows deposition of IgG and complement.

- In The class III category there is evidence of membranoproliferative (MPGN), which is usually reported in whites. In the class IV category, there is evidence of focal proliferative/sclerosing lesion which is typically observed in blacks. Class III and IV categories contain most of the observed glomerular lesions in the patients who have been afflicted with schistosomal glomerulonephritis. They tend to occur most frequently in patients with significant fibrosis of the liver. A number of authors [51] [52] [71] [72] had reported frequent glomerular and peri-tubular IgA deposits these two classes. The afflicted patients tend to manifest with nephrotic syndrome, hypertension, and progressive impairment in the glomerular rate despite having had anti-parasitic treatment. [72] Lopes et al. [73] stated that the reason why blacks preferentially develop class IV sclerosing lesion is not clear, nevertheless, it could be related to genetic factors for example, polymorphism in APOL.
- In Class V category, there is amyloidosis of the kidney of the AA type which ensues from chronic inflammation as a result of persistent infection. Patients in the class V category, tend to manifest with significant proteinuria, including nephrotic syndrome, and reduced glomerular filtration rate. The course and outcome of this category tend to be similar to those associated with secondary amyloidosis of other causes/ [46]

- The suggested class VI category includes kidney disease associated with simultaneous schistosomal and hepatitis $C$ virus infection. [71] The kidney lesion in such patients includes mesangial proliferation, amyloidosis, fibrinoid necrosis, cellular apoptosis, and capillary thrombosis. All these features must be present simultaneously to be satisfy the criteria to belong to this category. Additionally immune complexes and cryoglobulins are deposited in the glomeruli. Barsoum [46] indicated that pathogenesis of this lesion is unclear, even though a complex interaction between the immune responses to shistosomiasis as well as hepatitis $\mathrm{C}$ had been postulated. The affected patients within this class are grossly hydronephrotic, hypertensive, severely undernourished, and they progress quickly to end stage renal failure (ESRF).

In addition to the five accepted categories and the proposed sixth category of schistosomal glomerulopathy, some authors [51] [52] [53] have pointed out that there are other patients with schistosomiasis as well as heavy proteinuria are observed to have membraneous nephropathy (MN) but this group of patients have not yet been included in the AFRAN classification as a result of the fact that so far there is lack of evidence for a pathogenic link.

\section{Diagnosis}

Barsoum [46] stated that a sequence of steps, are important in the process of the diagnosis of schstosomal glomerulopathy and that: The diagnosis commences or should be suspected: (a) with the clinical suspicion in a patient with kidney disease who is known or has been known to have schistosoma mansoni; (b) exposure to an endemic area, (c) clinical evidence / demonstration of chronic hepatosplenic schistosomiasis, (d) if the patient has not been diagnosed as having been afflicted with schistosomiasis, then schistosomal infection should be documented, (e) majority of patients with schistosomiasis and kidney disease should have kidney biopsy. (f) The patients should be evaluated for co-infection with salmonella, hepatitis $\mathrm{C}$ virus, and hepatitis $\mathrm{B}$ virus

\section{Barsoum [46] further stated that:}

- Even with the documentation of schistosomal infection and results of a renal biopsy which is consistent with schistosomal glomerulopathy, the diagnosis of schistosomal glomerulopathy is circumstantial and that a definitive diagnosis of schistosomal glomerulopathy, which is rarely obtained, necessitates the identification of parasitic antigens in the glomeruli.

- At the moment glomerular staining for schistosomal antigens is primarily a research technique in that it is only available in specialized laboratories.

Documentation of schistosoma infection

Barsoum et al. [46] intimated that:

- Schistosomal glomerulopathy occurs later on in the disease process and that stool and / or urine microscopy would only be rarely useful in the establishment of a diagnosis of schistosomiasis in patients with kidney disease. Instead, schistosomal infection is typically diagnosed in such patients 
by means of either serology or rectal biopsy or at times liver biopsy which had been indicated for a different reason.

- It has been documented that serological tests do identify schistosomal microsomal antigens (usually Mansoni Adult worm Microsomal Antigen [MAMA]) with the use of an enzyme-linked immuno-adsorbent assay (ELISA] [74] There is a corresponding ELISA assay which may be undertaken to detect Schistosoma haematobium (HAMA) and Schistosoma japonicum (JAMA) and serological assays for these antigens, if positive would suggest active infection, and hence serology would be considered the preferred diagnostic test where these tests are available.

- A large number of laboratories that are located in schistosomiasis endemic areas do not have the capability to perform the aforementioned serological tests; therefore, in such situations, a high-rectal sub-mucosal biopsy could be taken to demonstrate Schistosoma mansoni ova, old trapped eggs may be seen on histological examination and they tend to be calcified

The place of renal biopsy; and screening

Barsoum et al. [46] intimated that a biopsy of the kidney is usually indicated unless the kidney disease is benign. They stated further that:

- In a patient who manifests with mild proteinuria, for example, less than 1 gram per day, with or without haematuria, a normal glomerular filtration rate, and known schistosomiasis, biopsy of the kidney may be deferred. In such situations, the patients should be re-assessed pursuant to anti-parasitic treatment, and in the event of persistence of renal disease or progressive disease renal biopsy could be undertaken.

- Other patients who are presumed to have schistosomal glomerulopathy should have renal biopsy because biopsy of the kidney is helpful for classification which might influence the management options and might be useful in prognostication. Furthermore, biopsy of the kidney may be helpful in distinguishing other types of glomerular disease.

\section{Screening}

Barsoum et al. [46] iterated that considering the fact that patients who have been afflicted with hepatosplenic schistosomiasis and fibrosis of the liver have a high risk of developing glomerulopathy, even following effective anti-parasitic treatment, they would make the ensuing suggestions:

Such patients with no known kidney disease should be screened for kidney disease yearly with the use of (a) urine dipstick testing looking for proteinuria and haematuria and (b) serum creatinine. In the event of confirmation of an abnormal urine dipstick finding after repeat testing, the urine sediment must be examined and the proteinuria should be quantified by means of urine protein-to-creatinine ratio

\section{Differential diagnosis}

Barsoum et al. [46] stated that:

- The differential diagnosis of schistosomal glomerulopathy, include many primary and secondary glomerular diseases.

- The diagnosis of schistosomiasis glomeulopathy, tend to be made by the documentation of schistosomiasis in combination with consistent histological findings; nevertheless, the biopsy of the kidney, could indicate that the kidney disease is not due to schistosomiasis, for example, crescentic glomerulonephritis or membranous nephropathy $[\mathrm{MN}])$. Additionally, abnormal serological test results (for example, complement levels) tend to be uncommon in patients who have schistosomal glomerulopathy unless there is co-infection with salmonella, hepatitis B, pr hepatitis c virus.

Dash and Bhowmik [75] stated that:

- Schistosomal glomerulopathy may be confused with a diagnosis of cirrhotic glomerulopathy which is quite commonly characterized by mild non-visible haematuria and proteinuria; and it may also be confused with IgA nephropathy and both disorders, like schistosomal glomerulopathy, are characterized by glomerular deposition of IgA.

- Patients with cirrhotic glomerulopathy rarely, develop nephrotic syndrome, even though they tend to have liver disease. They seldom progress to end-stage renal disease (ESRD) and they tend not to have extra-hepatic manifestations of schistosomal disease. Furthermore, sub-endothelial IgA depositions occur in cases of cirrhotic glomerulopathy but they do not occur in schistosomal glomerulopathy.

Barsoum et al. [46] stated that even though IgA nephropathy can occur in nephrotic syndrome and ESRD, such patients do not tend to have liver fibrosis.

\section{Treatment and outcome}

Some authors [74] [76] had stated that the treatment of schistosomiasisassociated glomerular disease is pivoted around treatment of the underlying helminthic infection. Nevertheless, the clinical outcome varies according to the histological class of the disease. They also stated that immunosuppressive treatment is not warranted in patients with schistosomiasis-associated glomerular disease.

\section{Class I disease}

With regard to Class I disease, Barsoum [51] stated that the disease may remain sub-clinical, regress either spontaneously or with anti-parasitic therapy, or it may progress by transformation into other classes depending upon the presence of co-infections (for example, transformation into class II or VI), the presence of liver fibrosis (for example, transformation into class III or IV), presence of certain genetic polymorphisms

Mahmoud [77] stated that Class I Schistosomal glomerular disease, especially, in the presence of living ova in stools or rectal snip should be treated with praziquantel.

\section{Class II disease}

The treatment of class II disease has been summarized in the ensuing statements:

Class II disease is reversible with simultaneous or sequential treatment of the schistosomiasis by means of praziquantel medication and salmonella with antibiotics like ampicillin, trimethoprim-sulfamethoxazole as reported by Tsang and Wilson [74] or ciprofloxacin as reported by Bassily et al. [78]. Barnhill et al. [79] stated that treatment against salmonella alone would not be effective in view of the fact that these bacteria firmly attach to schistosomal worms and they hide under a protective layer of host proteins which covers the worms.

Other classes of disease (Class III, IV, and V)

Some authors [76] [80] stated that Class III, IV, and V disease progress relentlessly ending up in end-stage renal disease (ESRD) over a period of four to six years, irrespective of treatment. Barsoum [71] recommended that the schistosomiasis should be treated with an anti-parasitic medication, even though such treatment would unlikely lead to 
improvement in the renal disease and that immunotherapy drugs are not indicated for the treatment of these classes of the disease. Barsoum [71] also stated that:

Patients with class VI disease tend to go through a rapid clinical deterioration commonly, as a result of associated liver disease and severe hypoproteinemia.

A number of patients succumb to inter-current infection even prior to reaching end-stage renal disease.

It has been documented that Class VI glomerular disease is resistant to both anti-parasitic and antiviral therapy and clearance of hepatitis $\mathrm{C}$ viral infection improves difficult to achieve with the presence of schistosomal liver fibrosis. [81]

[B] Miscellaneous narrations and discussions from some reported cases, case series and review papers relating to Schistosomiasis associated glomerulopathy (glomerulonephritis and nephrotic syndrome

Neves et al. [82] stated that within Brazil, Schistosomiasis tends to be caused by Schistosoma Mansoni, which tend to occur within the North Eastern and South Eastern regions of the country. They additionally iterated that Schistosomiasis primarily, does affect the liver and the gastrointestinal tract, even though the kidneys could also be affected by the disease and this kidney affection by Schistosoma Mansoni has mainly been in the form of glomerulopathies. Neves et al. [82] described the characteristic features of patients who develop Schistosomiasisassociated glomerulopathies, including the management and kidney outcomes. Neves et al. [82] undertook a retrospective analysis of patients who had been diagnosed as having Schistosomiasis-associated glomerulopathy between 2002 and 2017. They evaluated the clinical, biochemical, as well as the histopathology examination finding data of the kidney biopsies of the patients. Neves et al. [82] summated the results as follows:

- $\quad$ Out of the 24 patients they had evaluated, 19 of the patients that amounted to $79.1 \%$ of the patients were male and 16 patients that amounted to $66.4 \%$ of the patients were white.

- $\quad$ The mean age of the patients was 38.58 years +/- 9.83 years.

- They had identified the hepato-splenic form of Schistosomiasis in 15 patients that amounted to $68.1 \%$ of the patients, nephroticnephritic syndrome in 13 patients that amounted to $54.1 \%$ of the patients, haematuria in 20 patients that amounted to $83.3 \%$ of the patients, and hypertension in 18 patients that amounted to $75.0 \%$ of the patients.

- Pathology examination of kidney biopsy specimens of individuals that had Schistosomiasis of the kidney demonstrated a predominance of membranoproliferative pattern in 17 patients that amounted to $70.8 \%$ of the patients.

- Immunohistochemistry staining studies of the kidneys of the patients who had Schistosomiasis associated kidney disease demonstrated that 19 of the patients that amounted to $82.6 \%$ of the patients had specimens of the kidney that exhibited immunoglobulin M (IgM) expression, 10 of the patients' kidney biopsy specimens that amounted to $43.4 \%$ of the patients demonstrated $\operatorname{IgM}+\operatorname{IgG}$ expression, as well as 1 that amounted to $4.3 \%$ of the cases showed a "full house" pattern.

- $\quad$ The median follow-up time was noted to be 59.70 months and by the end of this follow-up period, 9 patients that amounted to $37.5 \%$ of the patients did develop end-stage renal disease (ESRD). The baseline serum creatinine level was observed to be higher with regard to the patients who had developed ESRD in comparison with those patients who did not develop ESRD $(1.99+/-1.08$ versus $1.34+/-0.46 \mathrm{mg} / \mathrm{dL}, \mathrm{p}=0.05)$.

Neves et al. [82] concluded that their study represents one of the uncommon clinical studies related to Schistosomiasis-associated glomerulopathy with long follow-up and renal endpoints, which had shown that one third of their patients, independent of their histological form, did progress to dialysis.

Gonçalves et al. [83] reported a 36-years-old patient, brown, who had classical symptoms of nephrotic syndrome that included proteinuria greater than 3.5 grams per 24 hours, hypoalbuminemia, and hypercholesterolemia. Nevertheless, in view of a 7-years antecedent history of hepatosplenic history and portal hypertension, he did undergo a biopsy of his kidney and pathology examination of the biopsy specimens demonstrated $\operatorname{IgA}$ deposits within the mesangial layer and which were more intense than immunoglobulin $\mathrm{G}$ (IgG), that was accompanied by C1q and C3, with 4 out of 13 glomeruli sclerotic, standard light mesangial glomerulonephritis renal injury with $\operatorname{IgA}$ deposits. The patient was commenced on angiotensin receptor blocker (ARB) which resulted in progressive improvement with regards to the proteinuria. Gonçalves et al. [83] stated the ensuing:

- Kidney involvement is a severe form of Schistosomiasis which tends to occur in $10 \%$ to $15 \%$ of individuals who are afflicted by hepatosplenic form of Schistosomiasis.

- Nephrotic syndrome is the commonest clinical manifestation of the disease. Kidney involvement in Schistosomiasis represents a complication that is caused by immune complexes (IC), and it is uncommon to occur within the Brazilian context with a immunoglobin A (IgA) deposits.

- When installed, the kidney injury by Schistosoma Mansoni, does classically manifest as membranoproliferative glomerulonephritis, (mesangiocapillary) with lobular accentuation.

Gonçalves et al. [83] concluded that:

- Patients who have glomerulonephritis due to Schistosomiasis do not tend to show improvement of disease progression with anti-parasitic treatment.

- Nevertheless, the antiproteinuric therapy could slow down the progression of end stage kidney disease.

Dos-Santos et al. [84] made the ensuing summations related to Schistosomiasis of the kidney:

- Distinct patterns of glomerular lesions with the inclusion of membranoproliferative glomerulonephritis as well as focal segmental sclerosis tend to be associated with infection due to Schistosoma Mansoni, or Schistosoma Japonicum.

- $\quad$ Evidence does exist which has suggested immune complex deposition is the major mechanism that underlies the formation of the different forms of Schistosoma glomerulonephritis and which had also suggested that the immune complex deposition could be intensified by portal hypertension.

- The relationship that exists between focal segmental glomerulosclerosis and Schistosomiasis has remained to be poorly understood. 
- A clinicopathology classification of Schistosoma glomerulonephritis was proposed by the Pan African Association of Nephrology.

- Within Brazil, mass therapy of patients with utilization of oral medicaments had led to a decrease with regard to the occurrence of Schistosomiasis glomerulopathy.

- With regard to a survey of kidney biopsies that had been undertaken in Salvador, Brazil, from 2003 to 2009, only 24 patients that amounted to $4 \%$ of the patients that had undergone kidney biopsies were demonstrated to be positive for Schistosoma Mansoni infection. Out of these patients, only one patient had hepatosplenic form of the disease. Focal segmental glomerulosclerosis was identified in seven of the patients as well as membranoproliferative glomerulonephritis was identified with regard to four patients.

- $\quad$ Even though retrospective studies related to the prevalence of kidney diseases based upon biopsies of the kidney could be influenced by many patient selection biases, a change with regard to the distribution of glomerulonephritis associated with nephrotic syndrome was found together with a decline with regard to the occurrence of severe forms of Schistosomiasis.

Pallangyo et al. [85] reported a case of massive bilateral hydroureteronephrosis as well as end-stage renal disease which had resulted from chronic Schistosomiasis in a 38-years-old man of African origin. The 38-year-old man was a rice farmer of African origin who had manifested with elevated blood pressure, swelling of his abdomen, as well as reduced urinary output over the preceding 10 months. He had abdominal examination which demonstrated an intra-abdominal mass which had measured $30 \mathrm{~cm} \mathrm{x} 17 \mathrm{~cm}$ extended from his right hypochondrium downward to his right inguinal region outward to his umbilicus and crossing the midline. The results of his routine biochemistry and haematology blood test results revealed an estimated glomerular filtration rate of $3.9 \mathrm{~mL} / \mathrm{min}$, haemoglobin of 6.78 grams / decilitre, as well as many electrolyte abnormalities. He had computed tomography (CT) intravenous urography scan of abdomen which demonstrated hepatomegaly of $18 \mathrm{~cm}$, bilateral enlargement of kidney with hydroureteronephrosis, multiple calcifications within his urinary bladder. Schistosoma haematobium eggs were isolated examination of his rectal biopsy specimen which confirmed the diagnosis. Pallangyo et al. [85] stated that Schistosomiasis of the urinary tract could be distressing upon the urinary tract system in particular as well as the survival prospects of the patients generally. In view of this observation, it is important that an extensive evaluation of the genitourinary system should form a pivot for the timely diagnosis as well as prompt management of patients especially with regard to residents within Schistosoma-endemic communities who manifest with obstructive uropathy. Pallangyo et al. [85] also made the ensuing iterations:

- Globally, Schistosoma infections do affect more than 200 million individuals which does emanate in the loss of 70 million disability adjusted life years.

- Within the sub-Saharan Africa region, where greater than $85 \%$ of the global Schistosoma infections are found, it has been estimated that 120 million individuals tend to become symptomatic, and greater than 20 million individuals tend to have severe disease, and approximately 200,000 individuals die each year.
- Renal impairment represents a severe emanation of Schistosomiasis which does occur in approximately $6 \%$ of all individuals who have been infected by the Schistosomiasis and in $15 \%$ of those individuals who have the hepatosplenic form of Schistosomiasis.

Duarte et al. [86] stated that kidney involvement in Schistosoma Mansoni infection had not been well studied. Duarte et al. [86] investigated the occurrence of kidney abnormalities in patients who had hepatosplenic schistosomiasis (HSS), especially renal tubular disorders. Duarte et al. [86] undertook a cross-sectional study with 20 consecutive patients who had hepatosplenic schistosomiasis (HSS), who had been followed-up within a Medical Centre in Maceió, Alagoas, Brazil. They performed urinary acidification and concentration tests with utilization of calcium chloride $(\mathrm{CaCl} 2)$ after 12-hour period of water and food deprivation. They quantified within urine, the biomarker monocyte chemoattractant protein 1 (MCP-1). They calculated the fractional excretion of sodium $(\mathrm{FENa}+)$, trans-tubular potassium gradient $(\mathrm{TTKG})$, and solute-free water reabsorption ( $\mathrm{TcH} 20)$. The hepatosplenic schistosomiasis (HSS) group was compared to a group 17 healthy volunteers. Duarte et al. [86] summarized the results as follows:

- The mean age and gender of the patients were similar to those of the controls.

- Urinary acidification deficit was identified in $45 \%$ of the patients who had hepatosplenic schistosomiasis (HSS).

- Urinary osmolality was found to be significantly lower in patients who had hepatosplenic schistosomiasis (HSS) $(588+/-$ 112 versus $764+/-165 \mathrm{mOsm} / \mathrm{kg}, \mathrm{p}=0,001)$ after 12 -hour period of water deprivation.

- TcH20 was lower in patients who had hepatosplenic schistosomiasis (HSS) $(0.72+/-0.5$ versus $1.1+/-0.3, \mathrm{p}=0.04)$.

- Urinary concentration deficit was identified in $85 \%$ of patients who had hepatosplenic schistosomiasis (HSS).

- The values of MCP-1 were higher within the hepatosplenic schistosomiasis (HSS) group in comparison with the control group $(122+/-134$ versus $40+/-28 \mathrm{pg} / \mathrm{mg}-\mathrm{Cr}, \mathrm{p}=0.01)$ and this positively correlated with the values of microalbuminuria and proteinuria.

Duarte et al. [86] made the ensuing conclusions:

- Hepatosplenic schistosomiasis (HSS) is associated with important kidney dysfunction.

- The main abnormalities found included: urinary concentrating ability, and incomplete distal acidification defect, demonstrating the occurrence of tubular dysfunction.

- There was additionally, an increase in urinary MCP-1, which did appear to be a more sensitive marker of kidney damage than urinary albumin excretion rate.

Arndts et al. iterated the following summations related to some aspects of Schistosomiasis: [87]

- Within Sudan infections with Schistosoma Mansoni do remain a major health problem, where endemic communities including those within Kassala and Khartoum states do continue to face severe social-economic difficulties.

- Their previous immune-epidemiological findings that demonstrated different immune [cytokine and S. mansoni egg (SEA) antibody] profiles within individuals who had active 
infections with evidence of Schistosoma eggs within their stools that amounted to 110 patients, individuals who had been found to be positive for S. Mansoni via utilization of polymerase chain reaction (PCR) with the use of sera (SmPCR+ that consisted of 63 cases) and those individuals who were uninfected (Sm uinf).

- As antibody responses to eggs and worms are known to change during the period of infection, they had expanded the profiling further by the determination of levels of the adult worm (SWA) antibodies and nine chemokines within the serum of each individual in the three different cohorts. With the exception of C-C motif chemokine ligand (CCL)2, all of the measured chemokines were found to be significantly higher in SmPCR+ individuals in comparison with the egg+ group and in addition they also had presented levels of SWA-specific immunoglobulin (Ig)G2. Multi-variable regression analysis further did reveal that infection per se had been strongly linked to SWA-specific IgG3 levels and CCL5 was strongly associated with a SmPCR+ diagnostic state. In the absence of PCR diagnostics which recognize juvenile worms or Schistosomulae motives, the identification of schistosome-specific traits should provide better into the understanding of the current prevalence rates of Schistosomiasis within schistosomiasis endemic communities, and by so doing, do take into account PCR+ nonegg+ individuals within the current treatment programmes.

It would be argued that the aforementioned summation has illustrated advances that have been made with regard to the diagnosis of schistosomiasis which would further help with regard to the early diagnosis of schistosomiasis related nephropathy.

Seck et al. [88] reported a 10 year-old boy who was admitted into the nephrology department who had manifested with generalized oedema, hypertension, as well as fatigue over the preceding 3 weeks. He did not have any significant past medical history and he had completed his immunization. His mother did report that he had had baths within stagnant water 5 years earlier in their village. He did not have any personal history or family history of haematuria or oedema. He was found on examination to have a height of $130 \mathrm{~cm}$, and he had weighed 30 kilograms, his temperature was 37 degrees centigrade, his blood pressure was 150/90 $\mathrm{mm} \mathrm{Hg}$ which was high, he had tachycardia with a heart rate of 100 beats per minute, he had oedema over his face, back, limbs, as well as mild ascites. His Cardiac and pulmonary auscultations were normal. His examination did not demonstrate any evidence of arthritis, or abnormality of his skin. The results of his haematology blood tests were reported as follows (Blood parameter): Red blood cells were 3.4 × $10^{12} / \mathrm{L}$; Haemoglobin 9 grams per decilitre; Mean corpuscular volume 85 femtoliters; white blood cells $7.8 \times 10^{9} / \mathrm{L}$; Eosinophil count $0.15 \times 10^{9} / \mathrm{L}$;
Platelet count 300 x 109/L; Blood urea nitrogen 73 mg / dL; Serum creatinine $1.8 \mathrm{mg} / \mathrm{dL}$; Estimated glomerular filtration rate (using original Schwartz formula) $40 \mathrm{~mL} / \mathrm{min}$; serum Calcium $7.9 \mathrm{mg} / \mathrm{dL}$; phosphoremia $6.0 \mathrm{mg} / \mathrm{dL}$; Erythrocyte sedimentation rate $45 \mathrm{~mm} / \mathrm{hr}$; Creactive protein $4.0 \mathrm{mg} / \mathrm{L}$; serum protein 45 grams / litre; serum albumin 20 grams / litre. The results of his urinalysis were reported as follows: Proteinuria $254 \mathrm{mg} /$ kilogram / day; Haematuria 23/ $\mathrm{mm}^{3}$; Leucocytes in urine $10 / \mathrm{mm}^{3}$; Urine culture - sterile; Schistosoma eggs - absent. The serology examination results of his hepatitis $\mathrm{B}, \mathrm{C}$, and HIV tests were negative and his serum complement (CH50) were slightly low. Cryoglobulins, and anti-DNA as well as anti-streptococcal antibodies were not found in his laboratory tests. He had ultrasound scan of the kidneys and lower urinary tracts that were found to be normal. A diagnosis of nephrotic syndrome was rendered and he was treated with utilization of oral prednisolone of $2 \mathrm{mg}$ per kilogram per day which was combined with captopril of $50 \mathrm{mg}$ per day, as well as salt-free diet, furosemide of $60 \mathrm{mg}$ per day, and oral supplementation with potassium, calcium, as well as vitamin D3. Four weeks subsequently, his oedema had decreased slightly as well as his urine albumin excretion did slow down at $187 \mathrm{mg} / \mathrm{kg} /$ day. His urine sediment was still not remarkable. He received a second 4 -week period of oral prednisolone which was started pursuant to three daily intravenous pulses of methylprednisolone $10 \mathrm{mg}$ per kilogram per day. Nevertheless, the results of his clinical and biological findings had not improved at the end of this treatment. $\mathrm{He}$ therefore underwent kidney biopsy and pathology examination of the biopsy specimen demonstrated type 1 membranoproliferative glomerulonephritis (MPGN) (see figure 2) with mild tubulointerstitial fibrosis and a granuloma that consisted of histiocytes and giant cells encompassing an egg of Schistosoma Haematobium (see figure 3). Congo red staining of the specimen was negative and immunofluorescence did not reveal any deposition. The results of his circulating IgG antibodies against Schistosoma antigens were high at 1 / 250. A final diagnosis of type 1 MPGN secondary to Schistosoma Haematobium was made. The histopathology examination findings of the kidney biopsy specimens did correspond to Class III-IV of the African Association Nephrology (AFRAN) classification. [71] [89]

Despite the absence of adult Schistosoma worms within the urine and the renal parenchyma of the patient, he received treatment that consisted on praziquantel with $40 \mathrm{mg} / \mathrm{kg}$ dose orally to ensure complete cure. After 1 month of this treatment, there was no improvement in the results of his renal function tests. Because of this, oral cyclophosphamide that comprised of $2 \mathrm{mg} /$ kilogram/ day was added to his corticosteroids with supportive treatment that comprised of salt-free diet, captopril, furosemide, calcium, as well as vitamin D3 for a period of 6 months. However, he did reach end-stage renal disease (ESRD) 1 year later and he had to be commenced on haemodialysis. 


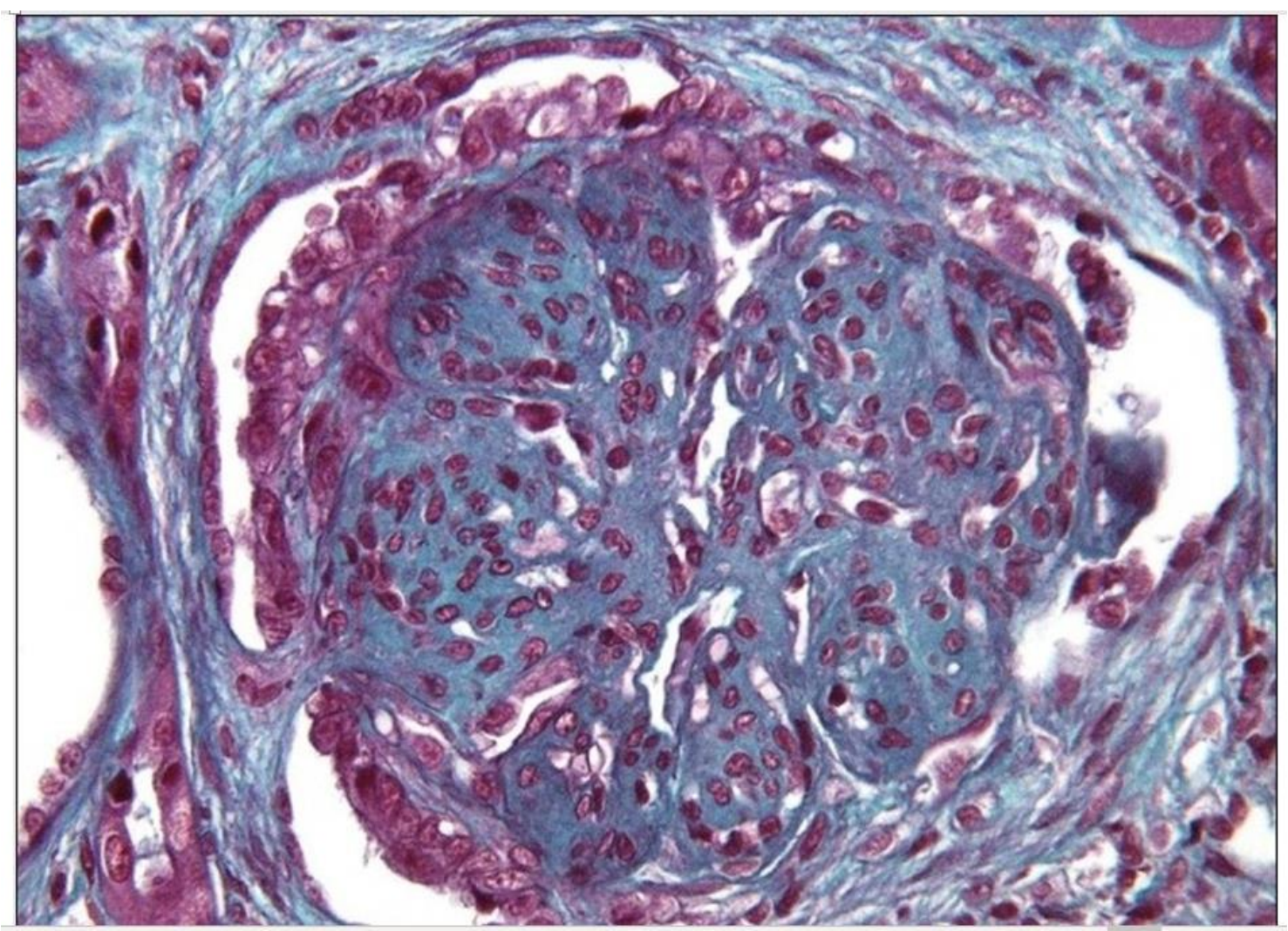

\section{Figure 2:}

Renal biopsy showing type 1 membranoproliferative glomerulonephritis with mild tubulointerstitial fibrosis (optical microscopy haematoxylineosin staining). Reproduced from: [88] Seck S M, Sarr M L, Dial M C, Ka E F. Schistosoma hematobium-associated glomerulopathy. Indian Journal of Nephrology 2011 Jul 01; 21(3): 201 - 203. DOI: 10.4103/0971-
$4065.78076 \mathrm{http}: / /$ europepmc.org/article/PMC/3161441 under copyright (C) Indian Journal of Nephrology. This is an open-access article distributed under the terms of the Creative Commons Attribution-NoncommercialShare Alike 3.0 Unported, which permits unrestricted use, distribution, and reproduction in any medium, provided the original work is properly cited.

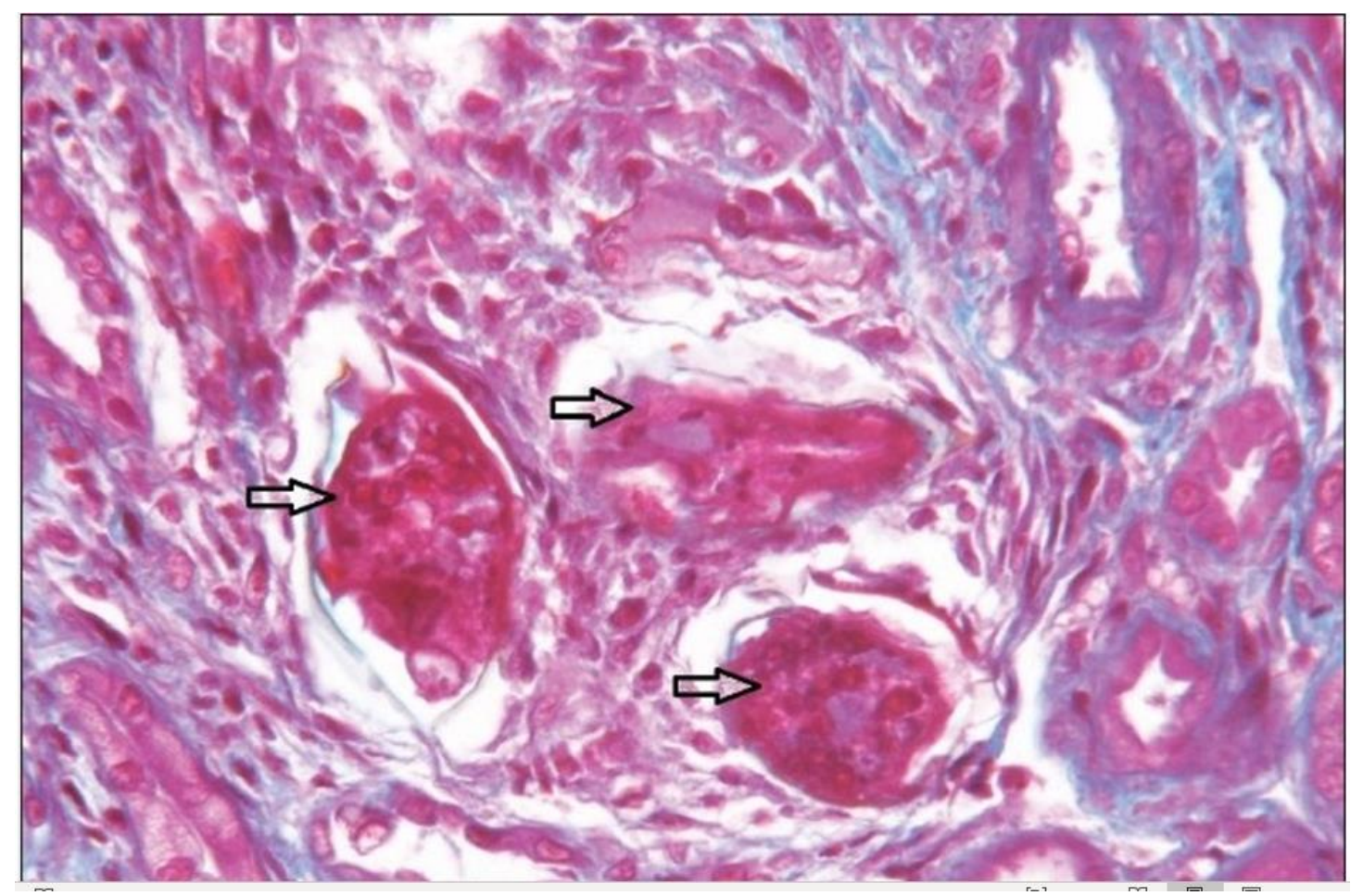

Figure 3. 
Granuloma made by histiocytes and giant cells surrounding three Schistosoma haematobium eggs (black arrows) PAS staining magnification x400. Reproduced from: [88] Seck S M, Sarr M L, Dial M C, Ka E F. Schistosoma hematobium-associated glomerulopathy. Indian Journal of Nephrology 2011 Jul 01; 21(3): 201 - 203. DOI: 10.4103/09714065.78076 http://europepmc.org/article/PMC/3161441 under copyright (C) Indian Journal of Nephrology. This is an open-access article distributed under the terms of the Creative Commons Attribution-NoncommercialShare Alike 3.0 Unported, which permits unrestricted use, distribution, and reproduction in any medium, provided the original work is properly cited.

Seck et al. [88] stated the following:

- Their reported case was the first case of Schistosoma Haematobium-associated MPGN to be reported in Senegal. Nevertheless, in relation to the high prevalence of Schistosomiasis within endemic areas, S. Haematobiumassociated MPGN could be underdiagnosed in view of the absence of systemic screening for schistosomiasis.

- In actual fact, MPGN is very uncommon among Senegalese patients. [90]

- With regard to their case, other causes of MPGN such as hepatitis $\mathrm{C}$ virus, as well as lupus had been excluded.

- Glomerular disease emanating from schistosomiasis had been reported in $10 \%$ to $15 \%$ of individual patients who had Schistosoma Mansoni infection and MPGN is the commonest histopathology examination pattern that had been documented by some authors. [51] [53] [71]

- Glomerular injury in Schistosoma associated nephropathy tends generally to be due to the direct deposition of Schistosoma antigens (GASP, gut-associated Schistosomal proteoglycan). Chronic hepatosplenic Schistosoma infections does play an important role with regard to the subsequent progression of the disease [51] [89]

- Schistosoma haematobium does have a greater trophism for the lower urinary tract including the ureter, urethra, and urinary bladder. Their reported case of MPGN-associated with Schistosoma Haematobium infection was not usual; nevertheless, the mechanism of glomerular deposition of immunofluorescence in contrast with MPGN related to Schistosoma Mansoni where glomerular and peri-tubular IgA deposits tend to be frequent as was documented by some authors. [51] [61 [71]

- With regard to the manifestation in their reported case, the case was that of a steroid-resistant nephrotic syndrome that was associated with increased serum level of creatinine.

- With regard to glomerular disease that is associated with Schistosoma Mansoni infections, the kidney manifestations could vary from asymptomatic haematuria and normal kidney function to end stage renal disease (ESRD). [71] Nevertheless, majority of patients tend to manifest with nephrotic syndrome, hypertension, and plasma concentration between 1 and 2 milligrams per decilitre ( $88 \mu \mathrm{mol} / \mathrm{L}$ and $176 \mu \mathrm{mol} / \mathrm{L}$ ). [80].

- The absence of haematuria and leukocyturia with regard to their patient was compatible with low disease activity even if the total serum complement was slightly consumed. The presence of mild fibrosis as well as some sclerotic glomeruli within the kidney biopsy specimen of their patient did suggest an advanced injury to the kidney by the disease. It is not known whether Blacks do preferentially develop focal segmental glomerulosclerosis in this setting of disease. Progression of the disease to End stage renal disease (ESRD) did occur quickly in their reported patient despite his treatment with aggressive treatment. Their observation relating to the sequence of events in their patient, had confirmed the bad prognosis of classes IIIIV in which treatment with utilization of both anti-helminthic medicaments and immunosuppressive medicaments (prednisolone, and cyclophosphamide) tends usually not to be effective in progression to End Stage Renal Disease (EDRD). [71] [91]

- Conversely, with regard to the first two classes of the disease, it has been iterated that complete recovery could occur spontaneously or pursuant to treatment. [71] [80]

- In order to reduce morbidity related to Schistosoma-associated nephropathy, public health policy, should be concentrated upon the prevention of the disease by the control of snail, improved sanitation, and health education, as well as by the implementation, and sustenance of chemotherapy-based control strategies. [91]

Seck et al. [88] made the ensuing conclusions:

- MPGN is an uncommon complication associated with Schistosoma Haematobium infection which tends to be associated with potentially poor prognosis.

- $\quad$ MPGN could lead quickly to End Stage Renal Disease (ESRD).

- Anti-helminthic and immunosuppressive medicaments tend not to be effective at advanced stages of the disease as well as efforts might focus upon the prevention, early detection, as well as treatment of Schistosoma infections among at-risk groups of individuals.

\section{Conclusions and Recommendations}

- Schistosomiasis is the second most devastating tropical parasitic disease globally which tends to be responsible for many urological complications. Nevertheless, glomerular injury is an uncommon complication which has mainly or most often described with Schistosoma Mansoni. However, an unusually rare case of membranoproliferative glomerulonephritis (MPGN) associated with Schistosoma haematobium infection in a young boy living in a rural area has also been reported.

- It has been illustrated that when patients who have Schistosomiasis-associated Kidney disease are managed and followed-up on long-term basis with renal end points, one third of the patients independent of the histopathology examination features of the kidney biopsies would tend to progress dialysis.

- The baseline serum creatinine level of the patients was observed to be higher with regard to the patients who had developed ESRD in comparison with those patients who did not develop ESRD.

- Hepatosplenic schistosomiasis (HSS) is associated with important kidney dysfunction.

- The main abnormalities found included: urinary concentrating ability, and incomplete distal acidification defect, demonstrating the occurrence of tubular dysfunction. 
- There was additionally, an increase in urinary MCP-1, which did appear to be a more sensitive marker of kidney damage than urinary albumin excretion rate.

- MPGN is an uncommon complication associated with Schistosoma Haematobium infection which tends to be associated with potentially poor prognosis.

- $\quad$ MPGN could lead quickly to End Stage Renal Disease (ESRD).

- Anti-helminthic and immunosuppressive medicaments tend not to be effective at advanced stages of the disease as well as efforts might focus upon the prevention, early detection, as well as treatment of Schistosoma infections among at-risk groups of individuals.

- In order to reduce morbidity related to Schistosoma-associated nephropathy, public health policy, should be concentrated upon the prevention of the disease by the control of snail, improved sanitation, and health education, as well as by the implementation, and sustenance of chemotherapy-based control strategies.

- Considering that that numerous individuals are found yearly to be inflicted by Schistosomiasis who tend to dwell in more rural parts of their countries where facilities for the undertaking of renal function tests and biopsies of the kidney tend not to be readily available, there is the likelihood that Schistosomiasisassociated nephropathies may be highly underdiagnosed globally.

\section{Conflict of Interest Declaration - None}

\section{Acknowledgements}

Acknowledgements to:

- Indian Journal of Nephrology for granting permission for reproduction of figures and contents of their journal article under copyright (C) Indian Journal of Nephrology. This is an open-access article distributed under the terms of the Creative Commons Attribution-Noncommercial-Share Alike 3.0 Unported, which permits unrestricted use, distribution, and reproduction in any medium, provided the original work is properly cited.

- The Center for Disease Control and Prevention for making available to readers and clinicians a figure related to the life cycle of their summations related to the causative agents of Schistosomiasis

\section{References}

1. Schistosomiasis, Fact Sheet No. 115; 2010 February; World Health Organization.

2. World health Organization Weekly epidemiological record 30 April No.18, 2010; 85: 157-164.

3. Chistulo L, Loverdie P, Engels D. (2004) Diseases Watch: Schistosomiasis. TDR Nature Reviews Microbiology.

4. Nawal M Nour N M. Schistosomiasis: Health Effects on Women. Reviews in Obstetrics \& Gynecology 2010 Winter; 3(1): $28-32$.

5. Ahmed S H, Cunha B A, John Jr J F, Talavera F. (2013) Schistosomiasis. Medscape.

6. Corachan M. (2002) Schistosomiasis and international travel. Clin Infect Dis. 35(4): 446-450.

7. Houston S, Kowalewska-Grochowska K, Naik S, McKean J, Johnson E S, Warren K. (2004) First report of Schistosomiasis mekongi: infection with brain involvement. Clinical Infectious Diseases. 38(1):1-6.

8. Mohammad A Z, Edino S T, Samaila A A. (2007) Surgical pathology of schistosomiasis. Journal of the National Medical Association. 99(5):570-574.

9. McCullough F S. (1962) Further observations on Bulinus (Bulinus) truncates rohlfsi (Clessin) Seasonal population fluctuations and biology. Bull World Health Organ Bulletin World Health Organization. 27(1): 161-170.

10. World Health Organization. Schistosomiasis 2020.

11. Lapa M, Dias B, Jardim C, Fernandes C J, Dourado P M, Figueredo M, Farias A, Tsutsui J, Terra-Filho M, Humbert M, Souza R. (2009) Cardiopulmonary manifestations of hepatosplenic schistosomiasis. Circulation. 19(11): 1518-1523.

12. Walker M, Zunt J R. (2005) Parasitic central nervous system infections in immunocompromised hosts. Clin Infect Dis. 40(7): 1005-1015.

13. Wan H, Lei D, Mao Q. Cerebellar schistosomiasis: a case report clinical analysis. Korean J Parasitol 2009 Mar; 47(1): 53-56.

14. Badmos K B, Komolate A O, Rotimi O. (2006) Schistosomiasis presenting as acute appendicitis. East Afr. Med J. 83(10): 528 532.

15. Terada T. (2009) Schistosomal appendicitis: incidence in Japan and a case report. World J Gastroenterol. 15(13): 1648-1649.

16. Argemi X, Camuset G, Abou-Bakar A, Lucescu I, Forestier E, Christmann D, Hansmann Y. (2009) Case report: rectal perforation caused by Schistosoma haematobium. Am J. Trop Med Hyg. 80(2):179-181.

17. Nmorsi O, Ukwandu N, Egmungenya O, Obhiemi N. (2005) Evaluation of $\mathrm{CD} 4(+) / \mathrm{CD} 8(+)$ status and urinary tract infections associated with urinary tract infections associated with urinary schistosomiasis among some rural Nigerians. Afr. Health Sci. 5(2): 126-130.

18. Coutinho H M, Acosta L P, Wu H W, McGarvey S T, Su L, Langdon G C, Jiz M A, Jarilla M, Olveda R M, Friedman J F, Kurtis J D. (2007) Th2 cytokines are associated with persistent hepatic fibrosis in human Schistosomiasis japonicum infection. Journal of Infectious Diseases. 195(2): 288-295.

19. Centers for Disease Control and Prevention (CDC) Parasites Schistosomiasis.

20. Wikipedia the free encyclopedia Schistosomiasis.

21. Chitsulo L, Engels D, Montresor A, Savioli L. (2000) The global status of schistosomiasis and its control. Acta Trop. Oct $23 ; 77(1): 41-51$.

22. Oliveira G C, Rodrigues N B, Romanha A, Bahia D. (2004) Genome and genomics of schistosomes. Canadian Journal of Zoology. 82:375-390.

23. Vos T, Flaxman A D, Naghavi M, et al. (2012) Years lived with disability (YLDs) for 1160 sequelae of 289 diseases and injuries 1990-2010: a systematic analysis for the Global Burden of Disease Study 2010 [published correction appears in Lancet. 2013 Feb 23;381(9867):628. AlMazroa, Mohammad A, Memish, Ziad A. Lancet. Dec 15;380(9859):2163-2196.

24. WHO Guidelines for the Safe Use of Waste Water, Excreta and Greywater, Volume 4 Excreta and Greywater Use in Agriculture (third edition). Geneva: World Health Organization.

25. The World Health Organization / Department of Control of Neglected Tropical Diseases Chitsulo L. Ed. Schistosomiasis: number of people treated worldwide in 2014 Weekly epidemiological record. 91;53-60.

26. Colley D G, Bustinduy A L, Secor W E, King C H. (2014) Human schistosomiasis. Lancet. 383(9936):2253-2264.

27. World Health Organization Malaria Fact Sheet. No. 94 
28. Pennington L F, Hsieh M H. (2014) The Immunology of Urogenital Schistosomiasis In: Immune Response to Parasitic Infections Immunity to Helminths and Novel Therapeutic Approaches. 93-124.

29. Thétiot-Laurent S A, Boissier J, Robert A, Meunier B. (2013) Schistosomiasis chemotherapy Angewandte Chemie. 52(31): 7936-7956.

30. Jordan P. (1986) Schistosomiasis. The St Lucia Project, Cambridge University Press. 40(3): 367.

31. Droz J-P, Carme B, Couppié P, Nacher M, Thiéblemont C. (Eds). (2015) Tropical Hemato-Oncology. Springer.

32. Jamieson B G M. Schistosoma Biology, Pathology and Control. $1^{\text {st }}$ edition CRC Press 2016.

33. Cheng M. (2014) Ancient parasite that still infects people found in 6,200-year-old skeleton. The Associated Press.

34. Kelsey Lucyk, Aleksandra Loewenau and Frank W. Stahnisch. (2016) The Proceedings of the 21st Annual History of Medicine Days Conference 2012 The University of Calgary Faculty of Medicine, Alberta, Canada. Cambridge Scholars Publishing 203 pages.

35. Nour N M. (2013) Schistosomiasis: Health Effects on Women. Reviews in Obstetrics \& Gynecology. 3(1): 28-32.

36. Mwanakasale V, Siziya S, Mwansa J, Koukounari A, Fenwick A. (2009) Impact of iron supplementation on schistosomiasis control in Zambian school children in a highly endemic area. Malawi Med J. 21(1): 12-18.

37. Danso-Appiah A, Olliaro P L, Donegan S, Sinclair D, Utzinger J. (2013) Drugs for treating Schistosoma mansoni infection. Cochrane Database Syst Rev.

38. TDR For research on diseases of poverty UNICEF - UNDP World Bank - WHO. Praziquantel dose confirmed. TDR News and Events 2014 News and Press Releases.

39. Brinkmann U K, Werler C, Traoré M, Doumbia S, Diarra A. (1988) Experiences with mass chemotherapy in the control of schistosomiasis in Mali. Trop Med Parasitol. 39(2):167-174.

40. Kramer C V Zhang F, Sinclair D, Olliaro P L. (2014) Drugs for treating urinary schistosomiasis (Review) The Cochrane Collaboration The Cochrane Database of Systematic Reviews. 8(8):1-101.

41. Xiao, S. Mefloquine, a new type of compound against schistosomes and other helminthes in experimental studies. Parasitol Res 2013; 112: 3723-3740.

42. Walker M D. (2018)Etymologia: Antimony. Emerging Infectious Diseases. 24(8):1601.

43. Mosunjac M B, Tadros T, Beach R, Majmudar B. (2003) Cervical schistosomiasis, human papilloma virus (HPV), and human immunodeficiency virus (HIV): a dangerous coexistence or coincidence? Gynecol Oncol. 90(1): 211-214.

44. Leutscher P D, Ramarokolo C E, Hoffmann S, Jensen J S, Ramaniraka V, Randrianasolo B, Raharisolo C, Migliani R, Christensen N. . Coexistence of urogenital schistosomiasis and sexually transmitted infection in women and men living in an area where schistosoma haematobium is endemic. Clin Infect Dis. 47(6): 775-782.

45. Friedman J F, Mital P, Kanzaria H K, Olds G R, Kurtis J D. (2007) Schistosomiasis and pregnancy. Trends Parasitol. 23(4):159-164.

46. Barsoum R S, Glassock R J, Forman J P. (2014) Schistosomiasis and glomerular disease.

47. Essat E, Osman R A, Ahmet K Y, Soothill J F. (1974) The association between Schistosoma haematobium infection and heavy proteinuria. Trans R Soc Trop Med Hyg. 68: 315.

48. Essat E, Tohamy M, et al. (1978) Immunopathological study of glomerulonephritis associated with Schristosoma haematobium infection. In: Proceedings of the International Conference on Schistosomiasis, Cairo. 625-628.

49. Soliman M, Abdel-Salam E, et al. (1987) Schistosomiasis haematobium glomerulopathy: a clinical or a pathological entity?. In Abstracts of the $10^{\text {th }}$ International Congress of Nephrology, London.

50. KDIGO. (2012) KDIGO Clinical Practice Guidelines for Glomerulonephritis Kidney Int. Suppl. 2(2): 209.

51. Barsoum R S. (1993) Schistosomal glomerulopathies. Kidney Int. 44(1):1-12.

52. Sobh M A, Moustafa F E, El-Housseini F, Basta M T, Deelder A M, Ghoneim M A. (1987) Schistosomal specific nephropathyleading to end-stage renal failure. Kidney Int. 31:1006-1011.

53. Nussenzveig I, De Brito T, Carneiro C R, Silva A M. (2002) Human Schistosoma mansoni-associated glomerulopathy in Brazil. Nephrology Dialysis Transplantation. 17(1): 4-7.

54. Houba V. (1979) Experimental renal disease due to schistosomiasis. Kidney Int. 16(1):30-43.

55. Hoshino-Shimizu S, De Brito T, Kanamura H Y, Canto A L, Silva A O, Campos A R, Penna D O, Da Silva L C. Human schistosomiasis: Schistosoma mansoni antigen detection in renal glomeruli. Trans R Soc Trop Med Hyg. 70(5-6):492-496.

56. Barsoum R S, Sersawy G, Haddad S, Hashem M B, Kamel M, Wassef N, Francis M, Ghonaimy E, Soliman M, Khashab O, et al. (1983) Hepatic macrophage function in schistosomal glomerulopathy. Nephrol Dial Transplant. 3(5):612-616.

57. Van Marck E A, Deelder A M, Gigase P L. (1977) Effect of partial portal vein ligation on immune glomerular deposits in Schistosoma mansoni-infected mice British Journal of Experimental. Pathology. 58(4):412-417.

58. Sobhy E, Ali M I, et al. (2001) B-lymphocyte subpopulations in schistosomal glomerulopathy. Med J Cairo Univ. 69: 507513.

59. Pearce E J, MacDonald A S. The immunobiology of schistosomiasis Nat Rev Immunol. 2(7):499-511.

60. Abdel-Badie I, Barsoum R S. (1999) B-lymphocyte immunoglobulin surface expression in schistosomal glomerulopathy Med J Ahmed Maher Hosp.

61. Barsoum R, Nabil M, Saady G, El-Kalioubi A, Iskander I, ElGarem A. (1996) Immunoglobulin-A and the pathogenesis schistosomal glomerulopathy. Kidney Int. Clinical Investigation. 50(3): 920-928.

62. Thomas M A, Frampton G, Isenberg D A, Shoenfeld Y, Akinsola A, Ramzy M, Lilleywhite J, Williams D G. (1982) A common Anti-DNA antibody idiotype and anti-phospholipid antibodies in sera from patients with schistosomiasis and filariasis with and without nephritis. J Autoimmun. 2(6):803811.

63. Hillyer G V. (1971) Deoxyribonucleic acid (DNA) and antibodies to DNA in the serum of hamsters and man infected with schistosomes. Proc. Soc. Exp. Biol. Med. 136(3):880-883.

64. Isnard A, Chevillard C. (2008) Recent advances in the characterization of genetic factors involved in human susceptibility to infection by schistosomiasis. Curr Genomics. 9(5):290-300.

65. McManus D P, Ross A G, Williams G M, Sleigh A C, Wiest P, Erlich A, Trachtenberg E, Guanling W, McGarvey S T, Li Y S, Waine G J. (2001) HLA class II antigens positively and negatively associated with hepatosplenic schistosomiasis in a Chinese population. Int J Parasitol. 31(7):674-680.

66. Hafez M H, Zaatar M, et al. (1991) HLA A1, B35, DR 1,4 and nephrotic syndromein adult Egyptians. Med J Cairo Univ. 
67. Barsoum R S. (1987) Schistosomal glomerulopathy: selection factors. Nephrol Dial Transplant. 2(6):488-497.

68. Barsoum R S, Bassily S, Baigh O K, Eissa M, El-Sheemy N, Affify N, Hassaballa A M. (1977) Renal disease in hepatosplenic schistosomiasis: a clinicopathological study. Trans R Soc Trop Med Hyg. 71(5):387-391.

69. Barsoum R S. (1999) Tropical parasitic nephropathies Nephrol Dial Transplant. 14(3):79-91.

70. Barnhill A E, Novozhilova E, Day T A, Carlson S A. (2011) Schistosomiasis-associated Salmonella resist antibiotics via specific fimbrial attachments to the flatworm. Parasit Vectors. 4:123.

71. Barsoum R. (2004) The changing face of schistosoma glomeulopathy Kidney Int. 66(6):2472-2484.

72. Martinelli R, Noblat A C, Brito E, Rocha H. (1989) Schistosoa mansoni-induced mesangiocapillary glomerulonephritis: influence of therapy. Kidney Int. 35(5):1227-1233.

73. Lopes A A, Port F K, James S A, Silveira M A, Martinelli R, Brito E, Rocha H. (2002) Race and glomerulonephritis in patients with and without hepatosplenic Schistosomiasis mansoni. Clin Nephrol. 58(5): 333-336.

74. Tsang V C, Wilkins P P. (1997) Immunodiagnosis of schistosomiasis. Immunol Invest. 26(1-2):175-188.

75. Dash S C, Bhowmik D. Glomerulopathy with liver disease patterns and management Saudi J Kidney Dis Transpl. 11(3): 414-420.

76. Ott B R, Libbey N P, Ryter R J, Trebbin W M. (1983) Treatment of schistosomiasis-induced glomerulonephritis. A case report and review of the literature Arch Intern Med. 143(7): 1477-1479.

77. Mahmoud A A. Praziquantel for the treatment of helminthic infections. Adv Intern Med. 32:193-206.

78. Bassily S, Farid Z, Barsoum R S, Soliman L A, Higashi G I, Miner W F. (1976) Renal biopsy in Schistosoma-Salmonella associated nephrotic syndrome. J T Med. 79(11):256-258.

79. Barnhill A E, Novozhilova E, Day T A, Carlson S A. (2011) Schistosoma-associated Salmonella resist antibiotics via specific fimbrial attachments to the flatworm. Parasit Vectors. 4:123.

80. Martinelli R, Noblat A C, Brito E, Rocha H. (1989) Schistosoma mansoni-induced mesangiocapillary glomerulonephritis: Influence of therapy. Kidney Int. 35(5):1227-1233.
81. Federal Bureau of Prisons Clinical Practice Guidelines. Evaluation and Treatment of Hepatitis $\mathrm{C}$ and Cirrhosis.

82. Neves P D M M, Jorge L B, Cavalcante L B, Malheiros D, Woronik V, Dias C B. (2020) Schistosomiasis-associated glomerulopathy: Clinical aspects, pathological characteristics, and renal outcomes. Clin Nephrol. 93(5):251-261.

83. Gonçalves F O, Fontes T M, Canuto A P. (2017) Schistosoma mansoni associated glomerulopathy with $\operatorname{IgA}$ mesangial deposits: case report. J Bras Nefrol. 39(1):86-90.

84. Dos-Santos W L, Sweet G M, Bahiense-Oliveira M, Rocha P N. (2011) Schistosomal glomerulopathy and changes in the distribution of histological patterns of glomerular diseases in Bahia, Brazil. Mem Inst Oswaldo Cruz. 106(7):901-904.

85. Pallangyo P, Bhalia S, Simelane N N, Lyimo F, Swai H J, Mkojera Z S, Hemed N R, Misidai N, Millinga J, Janabi M. (2020) Massive Bilateral Hydroureteronephrosis and EndStage Renal Disease Ensuing From Chronic Schistosomiasis: A Case Report. J Investig Med High Impact Case Rep.

86. Duarte D B, Vanderlei L A, Bispo R K, D A. Pinheiro M E, da Silva Jr. G B, Martins A M C, Meneses G C, Daher E D F. (2014) Renal function in hepatosplenic schistosomiasis--an assessment of renal tubular disorders. PLoS One.

87. Arndts K, Elfaki T E M, Jelden N, Ritter M, Wiszniewsky A, Katawa G, Goreish I A, Atti El Mekki M E Y A, Doenhoff M J, Hoerauf A, Layland L E. et al. (2019) Schistosoma mansoni $\mathrm{PCR}^{+}$-infected individuals in the Sudan present elevated systemic levels of chemokines when compared to uninfected and egg $^{+}$cohorts. Clin Exp Immunol. 196(3):364-373.

88. Seck S M, Sarr M L, Dial M C, Ka E F. (2011) Schistosoma hematobium-associated glomerulopathy. Indian Journal of Nephrology. 21(3):201-203.

89. Barsoum R S. (2003) Schistosomiasis and the kidney. Seminars in Nephrology. 23(1):34-41.

90. Abdou N, Boucar D, El Hadj Fary KA, Mouhamadou M, Abdoulaye L, Mourtala K A M, Saidou D, Abdoulaye P, Therese M D. (2003) Histopathological profiles of nephropathies in Senegal. Saudi J Kidney Dis Transpl. 14(2):212-214.

91. Gryseels B, Polman K, Clerinx J, Kestens L. (2006) Human schistosomiasis. Lancet. 368 (9541):1106-1118.

92. Technical report series. Vol. 912. Geneva: World Health Organisation; 2002. WHO Expert Committee, Prevention and control of schistosomiasis and soil-transmitted helminthiasis. 